\title{
Aerosol Science and Technology
}

\section{Accelerated Measurements of Aerosol Size Distributions by Continuously Scanning the Aerodynamic Aerosol Classifier}

Tyler J. Johnson, Jonathan P.R. Symonds, Jason S. Olfert \& Adam M. Boies

To cite this article: Tyler J. Johnson, Jonathan P.R. Symonds, Jason S. Olfert \& Adam M. Boies (2020): Accelerated Measurements of Aerosol Size Distributions by Continuously Scanning the Aerodynamic Aerosol Classifier, Aerosol Science and Technology, DOI: 10.1080/02786826.2020.1830941

To link to this article: https://doi.org/10.1080/02786826.2020.1830941

View supplementary material $匹$

Accepted author version posted online: 12

Oct 2020.

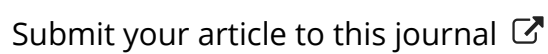

Q View related articles ¿ᄌ

View Crossmark data $₫$ 


\title{
Accelerated Measurements of Aerosol Size Distributions by Continuously Scanning the Aerodynamic Aerosol Classifier
}

Tyler J. Johnsona, Jonathan P.R. Symonds ${ }^{\mathrm{b}}$, Jason S. Olfertc, and Adam M.

Boies $^{a}$

aDepartment of Engineering, University of Cambridge, Cambridge, CB2 1PZ, U.K.

bCambustion Ltd., J6 The Paddocks, 347 Cherry Hinton Road, Cambridge, CB1 8DH, U.K.

cDepartment of Mechanical Engineering, University of Alberta, Edmonton, Alberta, T6G 2G8, Canada

Corresponding author: Tyler J. Johnson Email address: tjj31@cam.ac.uk

Shorten Running Title: Accelerated Measurements by Scanning the AAC

\begin{abstract}
Using an Aerodynamic Aerosol Classifier (AAC) upstream of a particle detector is a relatively new method for measuring the aerodynamic size distribution of an aerosol. This approach overcomes limitations of previous methodologies by leveraging the high transmission efficiency, independence from particle charging, and adjustable classification range and resolution of the AAC. However, the AAC setpoint must be stepped and stabilized before each measurement, which forces trade-offs between measurement time and step resolution. This study is the first to develop and validate theory which allows the speed of the AAC classifier to be continuously varied (following an exponential function), rather than stepped. This approach reduces measurement time, while increasing the resolution of the measured distribution. Assuming uniform axial flow, the transfer function of the scanning AAC and its inversion is determined. Limited trajectory theory is used to derive the idealized transfer function of the scanning AAC, while parameterized, particle streamline theory is used to develop the non-idealized transfer function, which accounts for nonidealized particle and flow behaviours within the classifier. This
\end{abstract}


theory and the practical implementation of the scanning AAC are validated by the high agreement of its measurements of polystyrene latex (PSL) particles (within $8.7 \%$ for six sizes between $100 \mathrm{~nm}$ to $2.02 \mu \mathrm{m}$ ), and of size distributions of three aerosol sources (Bis(2-Ethylhexyl) sebacate, $\mathrm{NaCl}$ and soot) to those measured by the stepping AAC (within $2 \%$ or better if the source stability is considered). The validity of assuming uniform axial flow in the classifier and downstream plumbing/detector are also discussed.

Keywords: accelerated classification, aerodynamic diameter, scanning transfer function, size distribution, aerosol characterization

\section{Introduction}

The size distribution is a fundamental property of an aerosol that commonly governs the behaviour of its particles. Since aerosol particles are often nonspherical, equivalent diameters, such as mobility or aerodynamic diameter, are used to describe them. The aerodynamic diameter $\left(d_{a}\right)$ is the equivalent diameter of a particle with the same settling velocity as a spherical particle with unit density $\left(\rho_{\mathrm{o}}=1000 \mathrm{~kg} / \mathrm{m}^{3}\right)$; while mobility diameter $\left(d_{\mathrm{m}}\right)$ is the equivalent diameter of a particle with same drag as a spherical particle when exposed to the same external force. These diameters are related to the relaxation time $(\tau)$ of the particle (i.e. time constant for the particle to reach its terminal velocity) by (Hinds, 1999):

$$
\tau=\frac{C_{c}\left(d_{\mathrm{a}}\right) \rho_{\mathrm{o}} d_{\mathrm{a}}^{2}}{18 \mu}=\frac{C_{c}\left(d_{\mathrm{m}}\right) \rho_{\mathrm{eff}} d_{\mathrm{m}}^{2}}{18 \mu},
$$

where $C_{c}$ is the Cunningham slip factor, $\mu$ is the surrounding gas viscosity and $\rho_{\text {eff }}$ is the effective density of the particle. The aerodynamic diameter describes the behaviour of the particle when its inertia dominates, such as deposition during inhalation (Finlay, 2001), settling in the atmosphere (ㅂinds, 1999) and separation by cyclones, impactors or filters (Kulkarni et al., 2011). Given these significant applications, various methodologies have been previously developed to measure the aerodynamic size distribution of an aerosol. 
Centrifuges (Hochrainer, 1971; Stöber and Flachsbart, 1971), impactors (Rao et al., 1992) and cascade impactors (Marple et al., 1991) can be used to measure the aerodynamic size distribution of an aerosol. However, these measurements are limited to one particle size at a time, or testing must be stopped to quantify the particles collected at each centrifuge location or impactor stage. To overcome these limitations, Keskinen et al. (1992) developed the Electrical Low Pressure Impactor (ELPI), which is a cascade impactor that quantifies the particles collected at each stage based on their electrostatic discharge upon collection. While this approach quantifies the aerodynamic size distribution in real-time, similar to other impactors, it requires high sample flow rates and low pressures to measure submicron particles. Furthermore, the size range and resolution of its measurements is limited by the number of impactor stages and their corresponding cutoff diameters. Recently, the resolution of the ELPI has been significantly improved through advanced inversion techniques (Saari et al., 2018). However, its measurements also depend on the unipolar charge distribution of the aerosol, which varies with particle size, morphology and composition (Biskos et al., 2004; Ouf and Sillon, 2009; Gopalakrishnan et al., 2013).

Others have developed methodologies for particle sizing based on their aerodynamic separation as the aerosol is expanded through a nozzle (Dahneke and Flachsbart, 1972). Kielser and Kruis (2017) used an aerodynamic lens (Liu et al., 1995) with a clean center sheath flow to classify particles based on their aerodynamic diameter. This system, referred to as the Differential Aerodynamic Particle Sizer (DAPS), is capable of measuring aerodynamic size distributions (between $80 \mathrm{~nm}$ and $3 \mu \mathrm{m}$ by changing its system pressure) in 4 minutes (Babick et al., 2018). Similar to the ELPI, the DAPS requires the particle charge distribution produced by its unipolar charger to be known and sufficient particle concentrations to generate detectable aerosol currents. Using an aerodynamic lens with a time-of-flight (TOF) chopper wheel to modulate the particle beam traveling over a known distance, Jayne et al. (2000) measured the vacuum 
aerodynamic diameter of particles in an Aerosol Mass Spectrometer (AMS). While AMS collects a large amount of particle characterization data (i.e. size, mass and chemical composition), these instruments are complex to operate. The Aerodynamic Particle Sizer (APS) spectrometer also uses TOF of particles, but between two intersecting laser beams downstream of a nozzle, to determine the aerodynamic size distribution of an aerosol (Wilson and Liu, 1980). However, the nozzle can break up or deform liquid particles due to the large velocity gradients it generates (Baron, 1986; Chen et al., 1990). While this methodology allows for near real-time characterization of an aerosol across a broad size range (from 500 $\mathrm{nm}$ to $20 \mu \mathrm{m}$ ), its measurements must be corrected 1 for particle density at larger sizes $(>5 \mu \mathrm{m})$ and densities $\left(>2 \mathrm{~g} / \mathrm{cm}^{3}\right)$ (Chen et al., 1990).

Alternatively, Mazumder and Kirsch (1977) measured the aerodynamic size distribution of an aerosol by exposing them to an acoustic excitation of a known frequency and calculating the phase shift between the medium and the suspended particles. The approach is referred to as the Single Particle Aerodynamic Relaxation Time (SPART) analyzer, and later evolved into the Electrical SPART (E-SPART) by replacing its oscillating acoustic field with an oscillating electrostatic field and charging the particles with a unipolar charger upstream of the analyzer (Renninger et al., 1981). However, both the SPART and E-SPART are limited to particles larger than $300 \mathrm{~nm}$ due to light scattering limitations as the particle size decreases (Renninger et al., 1981).

Stepping the setpoint of an Aerodynamic Aerosol Classifier (AAC) upstream of a particle detector can also be used to measure the aerodynamic size distribution of an aerosol (Johnson et al., 2018a). The AAC uses spinning, concentric cylinders and a sheath flow of clean gas to classify particles by their particle relaxation time (Tavakoli and Olfert, 2013). Unlike the aerodynamic methodologies previously described (such as the ELPI, DAPS, APS, and ESPART), the operating principle of the AAC allows its classification range and resolution to be easily adjusted (Tavakoli and Olfert, 2013). Furthermore, unlike 
the ELPI and DAPS, classifying particles using the AAC does not depend on their charge distribution (Tavakoli et al., 2014). The AAC can also classify particles as small as $25 \mathrm{~nm}$ (Cambustion, 2018), overcoming the minimum size the APS (i.e. $500 \mathrm{~nm}$ ) and SPART/E-SPART (i.e. $300 \mathrm{~nm}$ ) can characterize. Finally, the high transmission efficiency of the AAC allows aerosols with low particle concentrations (as low as tens of particles per $\mathrm{cm}^{3}$ with an appropriate particle detector) to be characterized (Johnson et al., 2018a, 2020).

Stepping the AAC to measure the aerodynamic size distribution of an aerosol leverages all of these advantages (Johnson et al., 2018a). However, its measurements are limited to when the AAC has stabilized at each setpoint. Thus, no measurements are collected while changing setpoints or during the time required for particles at the new setpoint to travel through the classifier and reach the detection region of the downstream particle detector. Therefore, collecting measurements with the stepping AAC takes significantly longer (minutes to tens of minutes) than the near real-time methodologies, such as the ELPI, APS, and E-SPART. This challenge of measurement time versus step resolution is similar to the one which prompted the evolution of the Differential Mobility Analyzer (DMA).

The DMA classifies particles by their electrical mobilities. This instrument also uses concentric cylinders and a sheath flow of particle-free air, but induces an electrostatic force on the particles (rather than a centrifugal force) by applying a potential difference between the cylinders (Knutson and Whitby, 1975). The DMA was first used to measure the electrical mobility size distribution of an aerosol by stepping its setpoint upstream of a particle detector (Knutson, 1976). This methodology is commonly referred to as the Differential Mobility Particle Sizer (DMPS). However, similar to the stepping AAC, this approach resulted in long measurement times and trade-offs with step resolution. To overcome this limitation, Wang and Flagan (1990) showed that continuously varying the electrostatic field of the DMA following an exponential function produces a mean 
field strength (i.e. average DMA voltage during the time a particle is in the classifier) that is equivalent to the field strength at steady-state. This approach forms the basis of the Scanning Mobility Particle Sizer (SMPS), an instrument that is widely used in aerosol research.

Others have built upon this original theory to further improve the accuracy, scan time or usability of the SMPS. For example, as the scans of the SMPS are accelerated the effect of viscous flow in the DMA classifier or in the downstream plumbing/detector become significant. Therefore, additional theory is required to account for the smearing of particle residence times (Collins et al., 2004; Mamakos et al., 2008; Dubey and Dhaniyala, 2008) and detection times (Russell et al., 1995; Collins et al., 2002; Kanaparthi et al., 2018). The effects of particle diffusion (Dubey and Dhaniyala, 2011; Huang et al., 2020) and entrance/exit geometry of the classifier (Mai and Flagan, 2018) on the transfer function of the SMPS have also been investigated. These studies have lead to the recent work of Mai et al. (2018) and Kanaparthi et al. (2018), which independently developed more advanced inversion theories to account for many of these effects on SMPS measurements.

Based on this evolution, this study develops the theory to continuously scan, rather than step, the AAC setpoint upstream of a particle detector. First, the SMPS theory developed by Wang and Flagan (1990) is expanded to the AAC to determine the required speed profile of its classifier. Assuming uniform axial flow of the gas in the classifier, the resulting transfer function of the scanning AAC based on limited trajectory theory $\left(\Omega_{\mathrm{sc}, \mathrm{LT}}\right)$ is derived, and compared to the corresponding solution of the steady-state AAC developed by Tavakoli and Olfert (2013). These results are then used to expand the experimental characterization of the steady-state AAC by Johnson et al. (2018a), which accounts for non-idealized particle and flow behaviours within the classifier, to the scanning AAC. This non-idealized transfer function of the scanning AAC ( $\Omega_{\mathrm{sc}, \mathrm{PS}, \mathrm{B}}$ ) is derived based on the particle streamline theory of the steady-state 
AAC operating with balanced classifier flows developed by Tavakoli and Olfert (2013). The average of these scanning transfer functions (i.e. $\bar{\Omega}_{\mathrm{sc}, \mathrm{LT}}$ and $\left.\bar{\Omega}_{\mathrm{sc}, \mathrm{PS}, \mathrm{B}}\right)$ over the counting time of the downstream particle detector is then determined. Finally, the deconvolution factors are derived to calculate the aerodynamic size distribution of the aerosol from the concentrations of the classified particles measured during scanning.

This scanning theory is validated by comparing the aerodynamic size distribution of three different aerosol sources (DOS [ Bis(2-Ethylhexyl) sebacate], salt and soot) measured by a scanning AAC to ones measured by a stepping AAC as previously developed by Johnson et al. (2018a). The scanning AAC is further validated by measuring polystyrene latex (PSL) particles of known sizes between $100 \mathrm{~nm}$ and $2.02 \mu \mathrm{m}$. Finally, the validity and effect of assuming uniform flow within the classifier and downstream plumbing/particle detector (i.e. neglecting the smearing of particle residence and detection times due to the flows being viscous) are discussed.

\section{Theory}

For clarity, the nomenclature used in this study is included as Section S1 of the Supplemental Information (SI). Furthermore for readability, only the notations required to differentiate multiple instances of the same parameter within the same section, table or figure of the article or SI are used. The title of a section, and captions/label of a table or figure are used to clarify the common instances of the parameter.

For example, consider the transfer function of the AAC denoted as $\Omega_{\mathrm{AAC}}$. If only one combination of instances is included within a section, such as the AAC operating at steady-state (ss) considering idealized (I), limited trajectory (LT) theory, the AAC transfer function within this section will be denoted as $\Omega_{\mathrm{AAC}}$ rather than $\Omega_{\mathrm{AAC}, \mathrm{ss}, \mathrm{LT}, \mathrm{I}}$. To avoid the ambiguity of this reduced notation, the title of this section (and also the footer if it is a SI section) would include steady-state 
(ss), idealized (I) and limited trajectory (LT). Please see SI Section S1.1 for further details.

\subsection{Radial Trajectories of Particles}

Starting from first principles, the centrifugal force field $\left(E_{\mathrm{c}}\right)$ at radial position $r$ within the AAC classifier that has angular speed $\omega$ at time $t$ can be calculated by:

$$
E_{\mathrm{c}}(t, r)=\omega^{2}(t) r
$$

This centrifugal force field induces a centrifugal force $\left(F_{\mathrm{c}}\right)$ on a particle proportional to its mass $m$ at radial position $r$ within the AAC classifier:

$$
F_{\mathrm{c}}=m \omega^{2}(t) r .
$$

However, the radial movement of the particle is counteracted by a drag force $\left(F_{\mathrm{d}}\right)$ from the surrounding gas molecules (Hinds, 1999):

$$
F_{\mathrm{d}}=\frac{1}{B} \frac{\mathrm{d} r}{\mathrm{~d} t}
$$

where $B$ is the mobility of the particle and $\mathrm{d} r / \mathrm{d} t$ is the velocity of the particle in the radial direction. Applying Newton's first law of motion in the radial direction and neglecting the radial acceleration of the particle? (i.e. $\mathrm{d}^{2} r / \mathrm{d} t^{2}=0$ ), the firstorder differential equation describing the radial velocity of a particle with relaxation time $r$ and radial position $r$ within the spinning classifier is:

$$
\begin{aligned}
& \sum F_{\mathrm{r}}=m \frac{\mathrm{d}^{2} r}{\mathrm{~d} t^{2}}=F_{\mathrm{c}}-F_{\mathrm{d}} \\
& \frac{\mathrm{d} r}{\mathrm{~d} t}=\tau \omega^{2}(t) r .
\end{aligned}
$$

This differential equation (Equation 5) can be solved as a separable equation for a particle at radial position $r_{\text {in }}$ at time $t_{\text {in }}$ which moves to radial position $r$ after time $t$. 


$$
\begin{gathered}
\frac{1}{\tau} \int_{r_{\text {in }}}^{r} \frac{1}{r^{\prime}} \mathrm{d} r^{\prime}=\int_{t_{\text {in }}}^{t_{\text {in }}+t} \omega^{2}\left(t^{\prime}\right) \mathrm{d} t^{\prime} \\
\tau=\frac{\ln \left(\frac{r\left(t_{\text {in }}+t\right)}{r_{\text {in }}\left(t_{\text {in }}\right)}\right)}{\int_{t_{\text {in }}}^{t_{\text {in }}+t} \omega^{2}\left(t^{\prime}\right) \mathrm{d} t^{\prime}}
\end{gathered}
$$

During steady-state operation, the angular speed of the AAC classifier is constant (i.e. $\omega(t)=\omega$ ) and the denominator of Equation 6 becomes the simple integration of a constant. However during scanning operation, additional considerations are required for the angular speed profile of the AAC classifier ( $\omega(t))$.

\subsection{Angular Speed Profile of AAC Classifier during Scanning}

To simplify the deconvolution of the transfer function of the scanning AAC, the angular speed profile of the AAC classifier $(\omega(t))$ must produce a proportional change in the centrifugal force field over time $t$, and the resulting critical particle trajectories it induces, that are independent of the times that the particles arrive at the classifier inlet $\left(t_{\text {in }}\right)$ as follows:

$$
\frac{E_{\mathrm{c}}\left(t_{\text {in }}+t, r\right)}{E_{\mathrm{c}}\left(t_{\text {in }}, r\right)}=i(t)
$$

where is a generic function that is only a function of $t$. This criteria is similar to that set by Wang and Flagan (1990) for the electrostatic force field generated by changing the voltage of a DMA classifier during SMPS operation. As derived in Section $\mathrm{S} 2$ for the scanning AAC, this independence from $t_{\text {in }}$ is achieved by the angular speed profile of:

$$
\omega^{2}(t)=\omega_{\mathrm{S}}^{2} \exp \left(\frac{t}{\tau_{\mathrm{sc}}}\right)
$$

as shown by the ratio of centrifugal fields (Equation 7), based on the definition of $E_{\mathrm{c}}$ (Equation 2), simplifying to: 


$$
\frac{E_{\mathrm{c}}\left(t_{\mathrm{in}}+t, r\right)}{E_{\mathrm{c}}\left(t_{\mathrm{in}}, r\right)}=\exp \left(\frac{t}{\tau_{\mathrm{sc}}}\right) .
$$

The time constant of the scan $\left(\tau_{\mathrm{sc}}\right)$, also derived in Section S2, is:

$$
\tau_{\mathrm{sc}}=\frac{t_{\mathrm{sc}}}{2 \ln \left(\frac{\omega_{\mathrm{E}}}{\omega_{\mathrm{S}}}\right)},
$$

where $\omega_{\mathrm{S}}$ and $\omega_{\mathrm{E}}$ are the initial and final angular speeds of the AAC classifier during the scan, respectively. Therefore, the AAC completing an up scan (i.e. $\omega_{\mathrm{E}}>\omega_{\mathrm{S}}$ ) is reflected in a positive scan time constant (i.e. $\tau_{\mathrm{sc}}>0$ ), while a down scan (i.e. $\omega_{\mathrm{E}}<\omega_{\mathrm{S}}$ ) is reflected in a negative scan time constant (i.e. $\tau_{\mathrm{sc}}<0$ ). An example of the required speed $(\omega(t))$ and acceleration $(\tilde{\omega}(t))$ profile for the AAC classifier for a $600 \mathrm{~s}$ scan from 20 to $700 \mathrm{rad} / \mathrm{s}$ is shown in Figure S2.1 of the SI.

Interestingly, it is also shown in Section S2 that $\omega^{p}(t)=\omega_{\mathrm{S}}^{p} C^{\frac{t}{\tau_{\mathrm{sc}}}}$ (where $p$ and $C$ are constants that are any real numbers, and $C$ must be greater than one) also satisfies $t_{\text {in }}$ independence (i.e. Equation 7). Furthermore, due to the relationship between $\omega(t)$ and $\tau_{\mathrm{sc}}$, the same angular speed profile of the AAC classifier is required regardless of the values of $p$ and $C$. However, for consistency with previous studies of the scanning DMA and the centrifugal force field generated in the AAC, this study uses $C=e$ and $p=2$ as shown in Equation 8.

\subsection{Minimum Scan Time}

The scan time $\left(t_{\mathrm{sc}}\right)$ of the AAC is limited by the angular acceleration/deceleration the classifier can achieve as a function of its angular speed (i.e. the acceleration/deceleration capacity of the classifier). As derived in Section S2.1, the required angular acceleration of the classifier $(\dot{\omega}(t))$ over a scan is linearly proportional to its required angular speed $(\omega(t))$ by a factor of $1 /\left(2 \tau_{\mathrm{sc}}\right)$. 
Therefore, as derived in Equations S2.15 and S2.16, the minimum scan time ( $\left.t_{\mathrm{sc}, \min }\right)$ the AAC can achieve is:

$t_{\mathrm{sc}, \min }=\frac{\omega(t)}{\dot{\omega}_{\max }(\omega(t))} \ln \left(\frac{\omega_{\mathrm{E}}}{\omega_{\mathrm{S}}}\right)$,

where for an up scan $\dot{\omega}_{\max }$ is the acceleration capacity of the AAC classifier at the end speed of the scan (i.e. $\omega(t)=\omega_{\mathrm{E}}$ ), while for a down scan $\dot{\omega}_{\max }$ is the deceleration capacity of the AAC classifier at the start speed of the scan (i.e $\left.\omega(t)=\omega_{\mathrm{S}}\right)$.

The minimum scan time varies slightly between different AACs due to variations in the acceleration/deceleration capacities of the classifiers caused by small differences in drive belt tension, as well as changes in bearing friction and motor efficiency over the lifetime of the instrument. To account for this variation, the scan time limitations of each AAC should be periodically updated by measuring the maximum acceleration and deceleration the classifier can achieve as a function of its angular speed. An example of these acceleration and deceleration capacities for multiple AACs and the variation between them is shown in Figure S2.2 of the SI.

\subsection{Uniform Axial Flow}

The radial position ( $n$ ) of a particle at time $t$ can be related to its axial position $(z)$ in the classifier by assuming the axial flow within the classifier is uniform (i.e. independent of its radial position). This assumption is commonly referred to as plug flow and the validity of this assumption is discussed in Section $\underline{4.2}$. Therefore, the axial velocity of the flow $(\mathrm{d} z / \mathrm{d} t)$ is treated as a constant $(\zeta)$ as follows:

$$
\frac{\mathrm{d} z}{\mathrm{~d} t}=U=\frac{Q_{\mathrm{a}}+Q_{\mathrm{sh}}}{\pi\left(r_{2}^{2}-r_{1}^{2}\right)}=\frac{Q_{\mathrm{sh}}}{\pi\left(r_{2}^{2}-r_{\mathrm{a}}^{2}\right)}=\frac{Q_{\mathrm{s}}}{\pi\left(r_{2}^{2}-r_{\mathrm{s}}^{2}\right)},
$$


where $Q_{\mathrm{a}}$ is the aerosol flow rate entering the classifier, $Q_{\mathrm{sh}}$ is the sheath flow rate entering the classifier, $Q_{\mathrm{s}}$ is the sample flow rate leaving the classifier, $r_{1}$ is the classifier inner radius, $r_{2}$ is the classifier outer radius, $r_{\mathrm{a}}$ is the classifier radius at the outer edge of the aerosol streamlines, and $r_{\mathrm{s}}$ is the classifier radius at the inner edge of the sample streamlines. These radii are illustrated in the simplified schematic of the AAC classifier geometry shown in SI Figure S3.1.

For plug flow, the particle residence time along the critical trajectory in the classifier $\left(t_{\mathrm{f}}\right)$ is independent of particle relaxation time $(t)$. Therefore, based on the axial velocity of the aerosol in classifier (Equation 12):

$t_{\mathrm{f}}=\frac{L}{U}=\frac{\pi L\left(r_{2}^{2}-r_{1}^{2}\right)}{Q_{\mathrm{a}}+Q_{\mathrm{sh}}}$

where $L$ is the length of the region that classifies the particles within the AAC classifier. The dimensions of the AAC classifier used in this study were $56 \mathrm{~mm}$, $60 \mathrm{~mm}$ and $206 \mathrm{~mm}$ for $r_{1}, r_{2}$ and $L$, respectively.

\subsection{Overall Trajectories of Particles assuming Uniform Axial Flow}

Assuming uniform axial flow and based on Equation 6, the relaxation time $(\tau)$ of a particle that migrates from $r_{\text {in }}$ to $r_{\text {out }}$ (i.e. the radial position of the particle at the classifier inlet and outlet, respectively) over its residence time in the classifier (i.e. $t=t_{\mathrm{f}}$, as defined by Equation 13) is:

$$
\tau=\frac{1}{K} \ln \left(\frac{r_{\text {out }}}{r_{\text {in }}}\right) \text {. }
$$

where:

$$
K=\int_{t_{\text {in }}}^{t_{\text {in }}+t_{\text {f }}} \omega^{2}\left(t^{\prime}\right) \mathrm{d} t^{\prime}
$$

During steady-state (ss) operation the classifier speed of the AAC is constant (i.e. $\omega(t)=\omega)$ and $K($ Equation 15) becomes: 
$K_{\mathrm{ss}}=\int_{t_{\mathrm{in}}}^{t_{\mathrm{in}}+t_{\mathrm{f}}} \omega^{2} \mathrm{~d} t^{\prime}=\omega^{2} t_{\mathrm{f}}$,

while during scanning (sc) operation, the classifier speed of the AAC $(\omega(t))$ continuously varies following Equation 8 and $K$ (Equation 15) becomes:

$$
\begin{aligned}
& K_{\mathrm{sc}}\left(t_{\mathrm{in}}\right)=\int_{t_{\text {in }}}^{t_{\mathrm{in}}+t_{\mathrm{f}}} \omega_{\mathrm{S}}^{2} \exp \left(\frac{t^{\prime}}{\tau_{\mathrm{sc}}}\right) \mathrm{d} t^{\prime} \\
& K_{\mathrm{sc}}\left(t_{\mathrm{in}}\right)=\omega_{\mathrm{S}}^{2} \tau_{\mathrm{sc}} \exp \left(\frac{t_{\mathrm{in}}}{\tau_{\mathrm{sc}}}\right)\left[\exp \left(\frac{t_{\mathrm{f}}}{\tau_{\mathrm{sc}}}\right)-1\right] .
\end{aligned}
$$

The time during the AAC scan can also be expressed as measurement time $\left(t_{\mathrm{m}}\right)$, which is the time the particles leave the classifier as follows:

$t_{\mathrm{m}}=t_{\mathrm{in}}+t_{\mathrm{f}}$.

Therefore, $K_{\mathrm{sc}}$ (Equation 17) can also be expressed in terms of $t_{\mathrm{m}}$ (defined in Equation 18) as follows:

$$
\begin{aligned}
& K_{\mathrm{sc}}\left(t_{\mathrm{m}}\right)=\int_{t_{\mathrm{m}}-t_{\mathrm{f}}}^{t_{\mathrm{m}}} \omega_{\mathrm{S}}^{2} \exp \left(\frac{t}{\tau_{\mathrm{sc}}}\right) \mathrm{d} t^{\prime} \\
& K_{\mathrm{sc}}\left(t_{\mathrm{m}}\right)=\omega_{\mathrm{s}}^{2} \tau_{\mathrm{sc}} \exp \left(\frac{t_{\mathrm{m}}}{\tau_{\mathrm{sc}}}\right)\left[1-\exp \left(\frac{-t_{\mathrm{f}}}{\tau_{\mathrm{sc}}}\right)\right] .
\end{aligned}
$$

\subsection{Idealized Transfer Function of AAC: Limited Trajectory and Uniform Axial Flow}

Limited trajectory theory is commonly used to estimate the transfer function of aerosol instruments, including the steady-state DMA ( $\underline{\text { Hoppel, }}$ 1978), scanning DMA (Wang and Flagan, 1990), and steady-state AAC (Tavakoli and Olfert, 2013). Therefore, this approach was used to derive the transfer function of the scanning $A A C$ assuming uniform axial flow, as shown in Section S3. Based on this derivation, the steady-state (Tavakoli and Olfert, 2013) and scanning AAC transfer functions have the same form (i.e. same shape) as summarized by the similar equations in Table 1 . The shape of the transfer function of the scanning 
AAC is also independent of the measurement time $\left(t_{\mathrm{m}}\right)$, but this result is only valid when uniform axial flow is a reasonable assumption (i.e. sufficiently long scan times) as discussed in Section 4.2. The shape of these transfer functions with balanced or unbalanced classifier flows is triangular or trapezoidal, respectively, as shown in Figure S3.2 of the SI. These shapes agree with those previously found by Tavakoli and Olfert (2013) for the steady-state AAC.

Therefore, the transfer function of the steady-state AAC has a constant shape and position in the particle relaxation time domain (i.e. AAC setpoint, $\tau^{*}$ ) as $K_{\text {ss }}$ (Equation 16) is a constant. The transfer function of the scanning AAC with a sufficiently long scan time also has a constant shape, but its setpoint $\left(\tau_{\mathrm{sc}}^{*}\right)$ varies as $K_{\mathrm{sc}}$ changes based on the time (i.e. Equations 17 or 19 for $t_{\mathrm{in}}$ or $t_{\mathrm{m}}$, respectively) the particles are classified during the scan.

An example illustrating these transfer function characteristics is shown in Figure 1 for the scanning AAC completing a 600 s up scan with balanced classifier flows ( $Q_{\mathrm{a}}=0.3 \mathrm{~L} / \mathrm{min}$ and $Q_{\mathrm{sh}}=3 \mathrm{~L} / \mathrm{min}$ ), and the steady-state AAC operating with the same parameters as those at the start and end of the scan. This figure also demonstrates the range of particle relaxation times measured by the scanning $A A C$ is smaller than measured by the steady-state AAC due to the residence time of the particles in the classifier $\left(t_{\mathrm{f}}\right)$. This effect is discussed in Section 2.9 and is expanded to also consider the effects of the counting time of the particle detector downstream of the scanning AAC.

However, the transfer function based on limited trajectory theory is an idealized representation of AAC classification. It neglects non-ideal particle behaviour within the classifier, such as diffusion and impaction, which affect the height (i.e. transmission efficiency) and width (i.e classification resolution) of the transfer function.

\subsection{Non-Idealized Transfer Function of AAC: Balanced Flows, Particle Streamline and Uniform Axial Flow}


To capture the non-ideal particle behaviour within the AAC, consider the outcome from the previous section that the steady-state and scanning AAC transfer functions have the same form (as summarized in Table 1) for sufficiently long scan times. Therefore, the transfer function of the steady-state AAC based on particle streamline theory with balanced (B) flow, developed by Tavakoli and Olfert (2013) and parameterized by Johnson et al. (2018a) to capture the nonidealized particle and flow behaviour, can also describe the scanning AAC. The adaption of this theory to the scanning AAC operating with balanced flows is shown in Section S4 and summarized in Table 2. For context, this table also shows the parallel parameters based on limited trajectory theory. Russell et al. (1995) used a similar approach to adapt the transfer function of the steadystate DMA developed by Knutson and Whitby (1975) based on particle streamline theory to the SMPS.

Similar to Johnson et al. (2018a), the transmission efficiency $\left(\lambda_{\Omega}\right)$ scales the area under the transfer function to account for particle losses, while the width factor $\left(\mu_{\Omega}\right)$ scales the full-width at half-maximum (FWHM) of the transfer function to account for broadening due to particle diffusion and other effects, such as the entrance and exit effects of the AAC classifier and non-idealized classifier flow. An example of the idealized and non-idealized transfer function based on particle streamline theory is shown in Figure S4.1 of the SI. The non-dimensional flow parameter $(\beta)$ controls the classification resolution of the AAC (i.e. FWHM of its transfer function) and is equivalent to (Tavakoli and Olfert, 2013):

$$
\beta=\frac{Q_{\mathrm{s}}+Q_{\mathrm{a}}}{Q_{\mathrm{sh}}+Q_{\mathrm{exh}}}
$$

where $Q_{\text {exh }}$ is the sheath flow rate leaving the classifier.

An example comparing the idealized and non-idealized representation of the transfer function of the scanning AAC operating with balanced classifier flows ( $Q_{\mathrm{a}}=0.3 \mathrm{~L} / \mathrm{min}$ and $Q_{\mathrm{sh}}=3 \mathrm{~L} / \mathrm{min}$ ) based on limited trajectory and particle 
streamline theory, respectively, is shown in Figure 2. The idealized transfer function based on particle streamline can also be represented by setting $\lambda_{\Omega}$ and $\mu_{\Omega}$ equal to 1 . This idealized representation predicts a transmission efficiency of one at the setpoint $\left(\tau_{\mathrm{sc}}^{*}\right)$ of the scanning AAC, while the comparable representation based on limited trajectory theory predicts less than one. This discrepancy in transmission efficiencies between the two theories is also observed in the corresponding transfer functions of the steady-state AAC, and is due to the particle streamline model neglecting the divergence of the centrifugal force field (Tavakoli and Olfert, 2013). However, this divergence is captured by the non-ideal parameters, $\lambda_{\Omega}$ and $\mu_{\Omega}$, which characterize the non-idealized transfer function of the AAC based on a parameterized model derived using particle streamline theory. Based on the values determined for these parameters by Johnson et al. (2018a), the non-idealized transferfunction of the scanning AAC also becomes shorter and broader as the aerodynamic diameter $\underline{3}$ setpoint ( $d_{\mathrm{a}, \mathrm{sc}}^{*}$ ) of the scanning AAC decreases from $3000 \mathrm{~nm}$ to $30 \mathrm{~nm}$, as shown in Figure $\underline{2}$.

\subsection{Average Transfer Function of Scanning AAC over Detector Time}

To determine the concentration of particles with relaxation time $r$ that pass through the AAC classifier at different measurement times $\left(t_{\mathrm{m}}\right)$, the change in the transfer function of the scanning AAC $\left(\Omega_{\mathrm{AAC}, \mathrm{sc}}\right)$ over the counting time of the downstream particle detector must be considered. Neglecting smearing effects of flow downstream of the AAC (as discussed later in Section 4.3), the average transfer function of the scanning AAC $\left(\bar{\Omega}_{\mathrm{AAC}, \mathrm{sc}}\right)$ over the counting interval $\left(t_{\mathrm{c}}\right)$ of the detector can be estimated by:

$$
\bar{\Omega}_{\mathrm{AAC}, \mathrm{sc}}=\frac{1}{t_{\mathrm{c}}} \int_{t_{\mathrm{m}}}^{t_{\mathrm{m}}+t_{\mathrm{c}}} \Omega_{\mathrm{AAC}, \mathrm{sc}}\left(\tau, t_{\mathrm{m}}{ }^{\prime}\right) \mathrm{d} t_{\mathrm{m}}{ }^{\prime} .
$$


This integral is solved for the idealized transfer function of the scanning AAC based on limited trajectory (i.e. Equation 27) in Section S5 and for the nonidealized transfer function of the scanning AAC with balanced flows based on particle streamline (i.e. Equation 28) in Section S6. These SI sections solve the integral by utilizing the Heaviside function $(\mathcal{H})$, defined as follows:

$$
\mathcal{H}(x-a)= \begin{cases}0 & \text { if } x-a<0 \\ 0.5 & \text { if } x-a=0 \\ 1 & \text { if } x-a>0\end{cases}
$$

to represent the mathematical discontinuities in the transfer function of the scanning AAC ( $\Omega_{\text {AAC,sc }}$ defined in Equations 27 or 28 ) in a single equation. The derivations also use the fact that the boundary conditions of $\Omega_{\mathrm{AAC}, \mathrm{sc}}$ are of the form:

$$
\tau_{x}=c_{\tau x} \exp \left(\frac{-t_{\mathrm{m}}}{\tau_{\mathrm{sc}}}\right)
$$

where $c_{\tau x}$ represents the grouped constants of boundary $x$ shown in Figures S3.2 and S4.1, and summarized in Tables S3.1 and S4.1 for the transfer function based on limited trajectory and particle streamline theory, respectively.

Based on this consistent form (i.e. Equation 36), the inequality relations based on particle relaxation time (i.e. $\tau>\tau_{x}$ ) used within the piecewise or Heaviside representation of the transfer function of the scanning $A A C$ are equivalent to:

$$
\tau>\tau_{x} \rightarrow \tau>c_{\tau x} \exp \left(\frac{-t_{\mathrm{m}}}{\tau_{\mathrm{sc}}}\right) \text {. }
$$

However, the isolation of $t_{\mathrm{m}}$ in this inequality (i.e. Equation 37) depends on the sign of $\tau_{\mathrm{sc}}$. If $\tau_{\mathrm{sc}}>0$,

$$
t_{\mathrm{m}}>\tau_{\mathrm{sc}} \ln \left(\frac{c_{\tau x}}{\tau}\right)
$$


while if $\tau_{\mathrm{sc}}<0$ (i.e. the inequality sign flips as dividing both sides by a negative number):

$t_{\mathrm{m}}<\tau_{\mathrm{sc}} \ln \left(\frac{c_{\tau x}}{\tau}\right)$

Based on these inequalities (i.e. Equations 38 and 39), the Heaviside function limits can be converted from the particle relaxation time $(\tau)$ to measurement time $\left(t_{\mathrm{m}}\right)$ domain as follows:

$$
\mathcal{H}\left(\tau-\tau_{x}\right)=\mathcal{H}\left(\frac{\tau_{\mathrm{sc}}}{\left|\tau_{\mathrm{sc}}\right|}\left(t_{\mathrm{m}}-\tau_{\mathrm{sc}} \ln \left(\frac{c_{\tau x}}{\tau}\right)\right)\right)
$$

Using this approach, the solutions for $\bar{\Omega}_{\mathrm{AAC}, \mathrm{sc}}$ (Equation 34) based on limited trajectory or particle streamline theory are summarized in Table $\underline{3}$, where the boundary conditions $T_{x}$ are determined using Equation 36 and the $t_{\mathrm{c}}$ shifted boundary conditions $\tau_{x, \mathrm{tc}}$ are determined as follows:

$$
\tau_{x, \mathrm{tc}}=c_{\tau x} \exp \left(\frac{-\left(t_{\mathrm{m}}+t_{\mathrm{c}}\right)}{\tau_{\mathrm{sc}}}\right)
$$

An example comparing the average transfer function of the scanning AAC ( $\left.\bar{\Omega}_{\mathrm{AAC}, \mathrm{sc}}\right)$ operating with balanced classifier flows $\left(Q_{\mathrm{a}}=0.3 \mathrm{~L} / \mathrm{min}\right.$ and $Q_{\mathrm{sh}}=3$ $\mathrm{L} / \mathrm{min}$ ) over three different counting times (i.e. $t_{\mathrm{c}}=0.1,1$ or $10 \mathrm{~s}$ ) of the downstream detector is shown in Figure $\underline{3}$. This figure shows both the idealized and non-idealized representations of the average transfer function for $600 \mathrm{~s}$ scans based on limited trajectory and particle streamline theory, respectively.

Figure $\underline{3} \mathrm{a}$ and $\underline{3} \mathrm{~b}$ show the average transfer functions at the start and end of the up scan, respectively, from 20 to $700 \mathrm{rad} / \mathrm{s}$ over $600 \mathrm{~s}$, and demonstrates that the shape of the idealized average transfer function based on limited trajectory theory does not change over the scan duration. However, the shape of the nonidealized average transfer function based on particle streamline theory does 
change over the scan duration to account for the changes in particle losses and transfer function broadening as the setpoint of the AAC (i.e. particle size of interest) changes.

Figure $3 \mathrm{c}$ shows the transfer functions at the start of the down scan from 700 to $20 \mathrm{rad} / \mathrm{s}$ over $600 \mathrm{~s}$. Therefore, during an up scan, the average setpoint of the scanning AAC (i.e. peak of the average transfer function denoted by $\bar{\tau}_{\mathrm{sc}}^{*}$ ) over $t_{\mathrm{c}}$ is less than the instantaneous setpoint of the scanning AAC $\left(\tau_{\mathrm{sc}}^{*}\right)$ at the start of the counting interval of the particle detector. Conversely, $\bar{\tau}_{\mathrm{sc}}^{*}$ is greater than $\tau_{\mathrm{sc}}^{*}$ during a down scan. This difference is due to the scanning AAC classifying increasingly smaller or larger particle relaxation times during an up or down scan, respectively. As expected, in all three examples, the average transfer function becomes broader and its amplitude lower as $t_{\mathrm{c}}$ increases due to the AAC scanning across a larger range of particle relaxation times.

\subsection{Parameters of Scanning AAC}

Similar to the average transfer function (i.e. Equation 34), the counting time of the particle detector also affects the average setpoint $\left(\bar{\tau}_{\mathrm{sc}}^{*}\right)$ and classes per decade (CPD) of the measurements collected by the scanning AAC. The CPD is the number of measurements collected across one decade of the parameter of interest. For the stepping or scanning AAC, the CPD corresponds to the number of amplitude measurements of the size distribution collected across one decade of particle relaxation time (e.g. 100 to $1,000 \mathrm{~ns}$, or $2,300 \mathrm{~ns}$ to $23,000 \mathrm{~ns}$ ). Therefore, as the CPD increases the change in particle size between consecutive measurements becomes smaller and characteristics of the size distribution are more clearly identified, such as multi-modal distributions. This parameter can be directly adjusted on the DMPS or stepping AAC by changing the number of setpoint steps across the size range of interest. However, for the scanning AAC or SMPS and maintaining the one-to-one correspondence 4 between the raw data and reported measurements, the maximum CPD is limited by the counting time of the downstream particle detector. 
The effects of counting time $\left(t_{c}\right)$ on these parameters of the scanning AAC can be quantified by solving the following equations:

$$
\bar{\tau}_{\mathrm{sc}}^{*}=\frac{1}{t_{\mathrm{c}}} \int_{t_{\mathrm{m}}}^{t_{\mathrm{m}}+t_{\mathrm{c}}} \tau_{\mathrm{sc}}^{*}\left(t_{\mathrm{m}}{ }^{\prime}\right) \mathrm{d} t_{\mathrm{m}}{ }^{\prime},
$$

and

$$
\mathrm{CPD}_{\mathrm{sc}}=\frac{n_{\mathrm{sc}}-1}{\left|\log \left(\bar{\tau}_{\mathrm{sc}, \mathrm{S}}^{*}\right)-\log \left(\bar{\tau}_{\mathrm{sc}, \mathrm{E}}^{*}\right)\right|},
$$

where $n_{\mathrm{sc}}$ is the number of measurements collected that are logarithmically spaced between the average setpoints of the AAC at the start and end of the scan (i.e. $\bar{\tau}_{\mathrm{sc}, \mathrm{S}}^{*}$ and $\bar{\tau}_{\mathrm{sc}, \mathrm{E}}^{*}$, respectively). These two equations are solved in Sections S7.1 and S7.2, respectively.

Furthermore, the range of particle relaxation times measured by the scanning AAC is smaller than the steady-state AAC due to the residence time of particles in the classifier ( $t_{\mathrm{f}}$ as defined in Equation 13). Measurements cannot be collected until the particles at the classifier inlet at the start of the scan pass through the it (i.e. $t_{\mathrm{m}}=t_{\mathrm{f}}$ ). Measurements can also only be collected for particles that experience the changing centrifugal force field during their entire residence time within the classifier. Therefore, measurements cannot be collected for particles at the classifier outlet after the scan duration (i.e $t_{\mathrm{m}}=t_{\mathrm{sc}}$ ). These limitations offset the start and end of AAC scan relative to its steady-state setpoint by $\bar{\tau}_{\mathrm{sc}}^{*} /\left.\tau_{\mathrm{ss}}^{*}\right|_{\mathrm{s}}$ and $\bar{\tau}_{\mathrm{sc}}^{*} /\left.\tau_{\mathrm{ss}}^{*}\right|_{\mathrm{E}}$, respectively, as derived in Section S7.3.

The solutions for these parameters of the scanning AAC (i.e. $\bar{\tau}_{\mathrm{sc}}^{*}, \mathrm{CPD}_{\mathrm{sc}}, \bar{\tau}_{\mathrm{sc}}^{*} / \tau_{\mathrm{ss}}^{*}$ Is and $\left.\bar{\tau}_{\mathrm{sc}}^{*} /\left.\tau_{\mathrm{ss}}^{*}\right|_{\mathrm{E}}\right)$ are summarized in Table $\underline{4}$. These equations are the same for either limited trajectory or particle streamline theory due to $\tau_{\mathrm{sc}}^{*}$ having the same form based on either theory (i.e. $c_{\tau^{*}} \exp \left(-t_{\mathrm{m}} / \tau_{\mathrm{sc}}\right)$ as summarized in Table $\underline{2}$ ). Based on these solutions (i.e. Table 4 ), the parameters for some example 5 AAC scans are shown in Table $\underline{5}$. This table demonstrates that a down scan can be 
completed quicker than an up scan due to the higher deceleration capacity of the $A A C$ relative to its acceleration as shown in Figure S2.2. However, as the scan time decreases the scan range becomes slightly narrower and the classes per decade (CPD) of the measurements collected decrease.

For context, a standard SMPS measurement using a 3080 Electrostatic Classifier with a 3081 DMA (TSI Inc., Shoreview, MN, USA) at low-flow (LF: $Q_{\mathrm{a}}=0.3 \mathrm{~L} / \mathrm{min}$ and $Q_{\mathrm{sh}}=3 \mathrm{~L} / \mathrm{min}$ ) completes an up scan in $120 \mathrm{~s}$ at $64 \mathrm{CPD}$, while measuring particles with electrical mobility diameters from 14 to $698 \mathrm{~nm}$. To scan across a similar size range in aerodynamic diameter (approximately 30 to $700 \mathrm{~nm}$ ), the minimum time of the up scan or down scan of the AAC ${ }^{5}$ at low-flow is 379 or 95 s, respectively. These parameters correspond to scans with 196 and 49 CPD, respectively. Therefore, completing a consecutive up and down scan with these parameters takes $474 \mathrm{~s}$, while completing only a down scan takes $-6235 \mathrm{~s}$. These up, down and combined scan times (379, 235 and 474 s, respectively) correspond to 3.2, 2.0 and 1.87 times longer than a standard SMPS measurement, while at a similar or higher CPD (64 vs. 49 or 196).

One key advantage of the scanning AAC is the broad range and upper limit of its particle sizing, scanning in tên minutes or less from approximately 30 to $2900 \mathrm{~nm}$ at low-flow and from approximately 200 to $6800 \mathrm{~nm}$ at high-flow (HF: $Q_{\mathrm{a}}=1.5$ $\mathrm{L} / \mathrm{min}$ and $Q_{\mathrm{sh}}=15 \mathrm{~L} / \mathrm{min}$ ). Shorter scan times are also possible if the scan range is focused on a smaller range of interest. For example, a portion of the PSL results shown in the Results (Section $\underline{4.1 .2}$ ) was collected using $60 \mathrm{~s}$ scans.

\subsection{Deconvolution of Average Transfer Function of Scanning AAC}

To determine the aerodynamic size distribution of an aerosol using a scanning AAC upstream of a particle detector, the particle concentration $\left(N_{\text {det }}\left(t_{i}\right)\right)$ that it classifies at time $t_{i}$ during the scan is determined by:

$$
N_{\text {det }}\left(t_{i}\right)=\int \eta(\tau) \bar{\Omega}_{\mathrm{AAC}}\left(\tau, t_{i}\right) \mathrm{d} N(\tau),
$$


where $\eta(\tau)$ is the counting efficiency of the downstream particle detector and $N(\tau)$ is the particle concentration of the aerosol source, both at particle relaxation time $\tau$. This relationship is similar to the methodology used to deconvolute measurements of the steady-state DMA (Stolzenburg and McMurry, 2008), scanning DMA (Wang and Flagan, 1990) or steady-state AAC (Johnson et al., 2018a). During scanning operation, $\bar{\Omega}_{\mathrm{AAC}}\left(\tau, t_{i}\right)$ is the average transfer function of the scanning AAC over the counting time of the particle detector at time $t_{i}$. During steady-state operation, the transfer function of the AAC is constant over the counting time of the particle detector. Therefore, the instantaneous $\left(\Omega_{\mathrm{AAC}, \mathrm{ss}}\right)$ and average $\left(\bar{\Omega}_{\mathrm{AAC}, \mathrm{ss}}\right)$ transfer function of the steady-state $\mathrm{AAC}$ are the same and Equation 50 becomes the same as that used by Johnson et al. (2018a) for the deconvolution of the steady-state AAC.

As shown in Section S8, Equation 50 can be expanded and rearranged to isolate the spectral density of the aerosol in terms of particle relaxation time (i.e. $\mathrm{d} N(\tau) / \mathrm{d} \log \tau$ ) as follows:

$$
\left.\frac{\mathrm{d} N(\tau)}{\mathrm{d} \log \tau}\right|_{\bar{\tau}_{\mathrm{sc}, i}^{*}}=\frac{N_{\mathrm{det}}\left(t_{i}\right) \ln (10)}{\eta\left(\bar{\tau}_{\mathrm{sc}, i}^{*}\right) \beta_{\mathrm{sc}, i}^{*}}
$$

where $\beta_{\mathrm{sc}, i}^{*}$ is the non-dimensional deconvolution parameter of the average transfer function of the scanning AAC at time $t_{i}$ as follows:

$$
\beta_{\mathrm{sc}, i}^{*}=\int \frac{\bar{\Omega}_{\mathrm{AAC}}\left(\tau, t_{i}\right)}{\tau} \mathrm{d} \tau
$$

This integral (Equation 52) is solved for the idealized, average transfer function of the scanning AAC based on limited trajectory theory (i.e. Equation 42) in Section S9 and for the non-idealized, average transfer function of the scanning AAC with balanced flows based on particle streamline theory (i.e. Equation 43) in Section S10. These solutions are also summarized in Table $\underline{6}$. The simple form of these solutions allows for direct implementation on the microprocessor of the $A A C$, thus allowing real-time data inversion and avoiding the need for an external 
computer during measurements. Similar to inversions for other aerosol classifiers, such as the DMPS (Knutson, 1976), SMPS (Wang and Flagan, 1990) and steady-state AAC (Johnson et al., 2018a), the inversion parameters for the scanning AAC were derived assuming the concentration of the aerosol is constant over the width of its average transfer function $\left(\bar{\Omega}_{\mathrm{sc}}\right)$. To avoid this assumption and also allow faster scans, future work could investigate the possibility of using higher-order inversion schemes, such as the Twomey algorithm used by Collins et al. (2002) or the L-curve algorithm used by Talukdar and Swihart (2003) or Kanaparthi et al. (2018) to improve the inversion of SMPS data.

It is interesting, but slightly counter-intuitive, that the deconvolution parameter based on particle streamline theory is the same for the scanning AAC (shown by Equation 54), as the one previously determined by Johnson et al. (2018a) for the steady-state AAC. As previously shown, the instantaneous transfer function of the steady-state $\left(\Omega_{\mathrm{AAC}, \mathrm{ss}}\right)$ and scanning $\left(\Omega_{\mathrm{AAC}, \mathrm{sc}}\right)$ AAC have the same shape (as summarized in Table 1 and shown in Figure 1), and thus the same area underneath them. Since these transfer functions (i.e. $\Omega_{\mathrm{AAC}, \mathrm{ss}}$ and $\Omega_{A A C, s c}$ ) are the same, the smearing of $\Omega_{\mathrm{AAC}, \mathrm{sc}}$ over to the counting time of the particle detector (i.e. resulting in $\bar{\Omega}_{\mathrm{AAC}, \mathrm{sc}}$ ) must maintain the same weighting of the average transfer function relative to particle relaxation time to achieve the same deconvolution parameter, as defined by Equation 52 . This fact is slightly counterintuitive and not immediately obvious by visual inspection. For example, the average transfer functions $\left(\bar{\Omega}_{\mathrm{AAC}, \mathrm{sc}}\right)$ shown in Figure $\underline{3}$ have deconvolution parameters (i.e. $\left.\int \bar{\Omega}_{\mathrm{AAC}, \mathrm{sc}} / \tau \mathrm{d} \tau\right)$ that are independent $\underline{8}$ of their counting times $\left(t_{\mathrm{c}}\right)$.

Figure 4 a shows that the deconvolution parameter $\left(\beta_{\mathrm{sc}}^{*}\right)$ based on idealized limited trajectory or particle streamline theory have excellent agreement (agree within $6.7 \%$ or less). Based on this idealized theory, the deconvolution parameter only depends on the dimensions (i.e. $r_{1}=56 \mathrm{~mm}, r_{2}=60 \mathrm{~mm}$ and $L=206 \mathrm{~mm}$ for the current commercial AAC) and flows (i.e. $Q_{\mathrm{a}}$ and $Q_{\mathrm{sh}}$, which are reflected in $\beta$ ) 
of the classifier. The effects of particle losses and transfer function broadening on the deconvolution parameter are shown in Figures $\underline{4 b}$ and $\underline{4} \mathrm{c}$ at two different samples flow rates ( $Q_{\mathrm{a}}=0.3$ or $1.5 \mathrm{~L} / \mathrm{min}$, respectively). These figures are based on particle streamline theory and use the non-idealized parameters previously determined by Johnson et al. (2018a) for the AAC. The white boundaries show the classification range of the current commercial version of the AAC based on the non-dimensional parameter of classifier flow ( $\beta$ as defined by Equation 33) and its average setpoint $\left(\bar{\tau}_{\mathrm{sc}}^{*}\right)$.

At the minimum sample flow of the AAC $\left(Q_{\mathrm{a}}=0.3 \mathrm{~L} / \mathrm{min}\right)$, the difference between the idealized $\left(\beta_{\mathrm{sc}, \mathrm{PS}, \mathrm{B}, \mathrm{I}}^{*}\right)$ and non-idealized $\left(\beta_{\mathrm{sc}, \mathrm{PS}, \mathrm{B}, \mathrm{NI}}^{*}\right)$ deconvolution parameter based on particle streamline theory increases with decreasing particle relaxation time, as shown in Figure $\underline{4 b}$. This trend agrees with expectations as the particle losses and transfer function broadening in the AAC increase as the particles become smaller (Johnson et al., 2018a).

At the maximum sample flow of the AAC $\left(Q_{\mathrm{a}}=1.5 \mathrm{~L} / \mathrm{min}\right)$, a portion of its classification range results in non-physical results for $\beta_{\mathrm{sc}, \mathrm{PS}, \mathrm{B}, \mathrm{NI}}^{*}$, as shown in Figure $4 \mathrm{c}$. This region produces non-physical results as the factor of $\beta / \mu_{\Omega}$ becomes greater than or equal to one, which implies the AAC transfer function spans to zero or negative particle relaxation times. This non-physical result is likely not due to the theory, but a result of extrapolating the non-idealized parameters previously determined by Johnson et al. (2018a) at $\beta=0.1$ to higher $\beta$ values. This inference is supported by the non-physical results starting at $\beta>0.46$ and the smallest particle size where $\mu_{\Omega}$ is the largest. This outcome highlights that the non-idealized parameters previously determined by Johnson et al. (2018a) should only be used at $\beta$ values close to 0.1 (as shown by the consistent results in Figure $\underline{4 b}$, which spans from $\beta=0.02$ to 0.15 ). Therefore, future work is required to characterize these non-idealized parameters at other flow conditions. 
Finally, the spectral density of the aerosol ( $\mathrm{d} N / \mathrm{d} \log \tau$ defined in Equation 51) can be converted to aerodynamic diameter $\left(\mathrm{d} N / \mathrm{d} \log d_{\mathrm{a}}\right)$ by the chain rule as shown in Section S8. This conversion requires the factor $\mathrm{d} \log \tau / \mathrm{d} \log d_{\mathrm{a}}$, which was previously determined by Johnson et al. (2018a) and included as Equation S8.9 in the SI for completeness.

\subsection{Effect of Delay Time Downstream of the AAC}

In addition to the residence time $\left(t_{\mathrm{f}}\right)$ of the particles in the AAC classifier, the time for the particles to travel from the classifier outlet to the downstream particle detector and be detected must also be considered. This time, henceforth referred to as the delay time $\left(t_{\mathrm{d}}\right)$, is defined as:

$t_{\mathrm{d}}=t_{\mathrm{t}}+t_{\mathrm{det}}$,

where $t_{\mathrm{t}}$ is the residence time or plumbing delay of the classified particles in the tubing between the classifier outlet and detector inlet, and $t_{\text {det }}$ is the response time of the particle detector, including the transport time of the particles within the detector. Since the equipment and its configuration downstream of the DMA in the SMPS configuration are the same as that downstream of the scanning AAC, the same theory developed for these effects in the SMPS can be applied. Assuming plug flow in the tube, $t_{\mathrm{t}}$ can be estimated by:

$t_{\mathrm{t}}=\frac{L_{\mathrm{t}}}{\bar{V}_{\mathrm{t}}}=\frac{\pi \mathrm{d}_{\mathrm{t}}^{2} L_{\mathrm{t}}}{4 Q_{\mathrm{s}}}$,

where $Q_{\mathrm{s}}$ is the sample volumetric flow rate of the AAC, while $L_{\mathrm{t}}$ and $\mathrm{d}_{\mathrm{t}}$ are the length and inner diameter of the tube, respectively, and $\bar{V}_{\mathrm{t}}$ is the average flow velocity within the tube. The validity of assuming uniform flow in the downstream tubing and particle detector is discussed in Section 4.3. However, it has been shown at sufficiently fast scan times in the SMPS, the viscous flow significantly smears the arrival times of the classified particles to the detection region of the downstream particle detector (Russell et al., 1995; Collins et al., 2002). 
While the delay time $t_{\mathrm{d}}$ for a given flowrate, tube length and particle detector can be estimated by calculating $t_{\mathrm{t}}$ (Equation 56) and based on the specifications of the detector, it is best to determine this value experimentally. The delay time can be determined by completing a bidirectional scan (i.e. consecutive up and down scan) of a stable, monodispersed aerosol source. If the delay time is correct, the measurements of the up and down scan will be the same. If the delay time is too short, the mode measured by the up scan will appear smaller in particle relaxation time/size than the down scan. While if the delay time is too long, the mode measured by the up scan will appear larger in particle relaxation time/size than the down scan.

This method is referred to as the up-down agreement (UDA) approach by Collins et al. (2004) and is one of three methods they used to determine the delay time of the SMPS. The transit time approach introduces a step-change in particle concentration and measures the time for it to travel through the system and be detected. The PSL approach introduces particles of known sizes (such as PSL particles) and shifts the measurements to the correct size by changing the delay time (Collins et al., 2004). Each of these three methods often provides different delay times, which propagate into the measurement error (Collins et al., 2004). This study only uses the UDA approach to determine the delay time for the scanning AAC, and its limitations are discussed further in Section $\underline{4.3}$.

Finally, the time $\left(t_{\mathrm{r}}\right)$ the particle detector reports its concentration measurement ( $\left.N_{\text {det }}\right)$ relative to the start of the scan $(t=0)$ is related to the measurement time of the scan $\left(t_{\mathrm{m}}\right)$ by:

$t_{\mathrm{m}}=t_{\mathrm{r}}-t_{\mathrm{c}}-t_{\mathrm{d}}$

where $t_{\mathrm{c}}$ is the counting time of the particle detector. As previously discussed in Section 2.9 (and also SI Section S7.3), the measurements collected during the AAC scan are only valid from $t_{\mathrm{m}}=t_{\mathrm{f}}$ to $t_{\mathrm{m}}=t_{\mathrm{sc}}$. 


\section{Experimental Setup}

The theory and practical implementation of the scanning AAC were validated by measuring PSL particles with known sizes. It was also validated by comparing the aerodynamic size distributions of three aerosol sources it measured to those measured by stepping the steady-state AAC, as previously developed by Johnson et al. (2018a). The experimental setup used to collect these measurements, using the AAC (Cambustion Ltd., Cambridge, UK) in stepping or scanning operation, is shown in Figure $\underline{5}$. For both measurement modes of the three aerosol sources, a 3775 Condensation Particle Counter (CPC; TSI Inc., Shoreview, MN, USA) was used as the particle detector. However, due to equipment availability, a 3752 CPC (TSI Inc.) was used as the particle detector for the PSL measurements. These detectors use the same operating principle (Agarwal and Sem, 1980), with the 3752 CPC being a newer version of the 3775 CPC.

For either stepping or scanning operation, the AAC controlled the CPC, logged its measurements at $1 \mathrm{~Hz}$ (i.e. detector counting time of $1 \mathrm{~s}$ ) and completed the inversion of the data in real-time. As developed by Johnson et al. (2018a), the steady-state AAC stepped its setpoint in aerodynamic diameter $\left(d_{\mathrm{a}, \mathrm{ss}}^{*}\right)$ and the corresponding concentration of the classified particles $\left(N_{\mathrm{det}}\left(d_{\mathrm{a}, \mathrm{ss}}^{*}\right)\right)$ was measured by the CPC. This approach limited its measurements to when the AAC had stabilized at a setpoint. Thus no measurements were collected while changing setpoints or during the time required for particles at the new setpoint to travel through the classifier and reach the detection region of the downstream particle detector. The scanning AAC continuously varied its average setpoint in aerodynamic diameter $\left(\bar{d}_{\mathrm{a}, \mathrm{ss}}^{*}\right)$ by following the required speed profile for the classifier (i.e. Equation 8). The corresponding concentration of the classified particles $\left(N_{\text {det }}\right)$ was measured by the CPC and logged by the AAC as a function of the reported time $\left(t_{\mathrm{r}}\right)$. This raw data was converted to a similar form as that from the steady-state AAC (i.e. $N_{\text {det }}\left(d_{\mathrm{a}}^{*}\right)$ ), by determining the measurement time ( 
$\left.t_{\mathrm{m}}\right)$ that corresponds to the reported time $\left(t_{\mathrm{r}}\right)$ using Equation 57, and then Equation 46 to determine the corresponding average setpoint of the scanning AAC $\left(\bar{\tau}_{\mathrm{sc}}^{*}\right)$. The equivalent aerodynamic diameter of this setpoint $\left(\bar{d}_{\mathrm{a}, \mathrm{sc}}^{*}\right)$ was determined using Equation 1.

The steady-state and scanning measurements were collected consecutively, with both an up and down scan completed in each measurement mode. This approach allowed the same equipment to be used for both measurement modes and thus avoided other sources of disagreement, such as the agreement between different AACs or CPCs, and different particle losses within the instruments or sample tubing. All of the measurements collected in this study used a $25 \mathrm{~cm}$ tube of conductive silicone with a $6 \mathrm{~mm}$ diameter between the AAC outlet and CPC inlet.

\subsection{Aerosol Sources}

The experimental setups used to generate the four different aerosol sources, DOS (Bis(2-Ethylhexyl) sebacate), $\mathrm{NaCl}$, soot and PSL particles, are shown in Figures $\underline{6}$ a to $\underline{6}$ d, respectively. The first three aerosol sources (i.e. DOS, $\mathrm{NaCl}$ and soot) were used to compare the measurements of the steady-state and scanning AAC. For all three of these aerosol sources, a rotating disk diluter (Cambustion Ltd.) was used to dilute the aerosol sample. The dilution ratios used for the DOS, $\mathrm{NaCl}$ and soot aerosol sources were 130, 200 and 350, respectively. These dilutions were selected to maintain the concentration of the classified particles at any AAC setpoint below $5 \times 10^{4}$ particles per $\mathrm{cm}^{3}$, thus increasing the accuracy of the 3775 CPC measurements by allowing it to operate in its single particle counting mode (TSI, 2007). The disk diluter was not used for the PSL measurements, as the concentrations of the particles classified by the scanning AAC were all within the higher single counting limit $\left(<1 \times 10^{5}\right.$ particles per $\left.\mathrm{cm}^{3}\right)$ of the $3752 \mathrm{CPC}(\underline{\mathrm{TSI}}, \underline{2017})$. 
Since the aerosol instruments only required 0.3 or $1.5 \mathrm{~L} / \mathrm{min}$ of sample flow, spills were used to exhaust excess flow. Aerosol sources generated with a liquid (i.e. DOS, $\mathrm{NaCl}$ and PSL) used liquid traps to remove excess liquid mobilized during atomization. Those sources where the liquid was distilled water (i.e. $\mathrm{NaCl}$ and PSL) also used a silica bead dryer to remove any water on the particles from atomization. To reduce the drying demand on the dryer, it was positioned after the spill on the diluted sample to minimize the particle concentration and flow rate passing through it.

Except for one size of PSL particles, the atomizer shown in Figure 6 a, b or $d$ was a Collison nebulizer (BGI Inc., Butler, NJ, USA) operating with HEPA-filtered air at 10 psig. A medicinal nebulizer (Aeroneb Go, Philips, Eindhoven, Netherlands) was used to produce the bigger droplets required to atomize the largest PSL size of $2.02 \mu \mathrm{m}$ (Figure $\underline{6} \mathrm{~d}$ ). The soot particles (Figure $\underline{6} \mathrm{c}$ ) were produced using a miniCast 4202 (Jing Ltd., Zollikofen, Switzerland) in autonomous mode (i.e. internal flow controllers) with the filtered air, filtered nitrogen and propane supplied at 2 bar. All of the aerosol sources were generated at positive pressure (i.e. above atmospheric pressure). However, due to the higher flowrates generated by the miniCast, a spill was required immediately downstream of its outlet. Therefore, the vacuum pump incorporated within the disk dilutor was used to pull a portion of the aerosol sample generated by the miniCast for dilution.

To limit the transient characteristics of the aerosol source affecting the agreement between the steady-state and scanning AAC results, each of the aerosol sources was operated for at least half an hour before collecting measurements. This warm-up time was increased to an hour for the miniCast. Furthermore, the external effects on the aerosol sources, such as temperature, were monitored, and pilot pressure regulators (11400-2G-PE100; Norgren $\mathrm{GmbH}$, Alphen, Germany) were utilized to improve the stability of the gas pressures used to generate all four aerosols shown in Figure $\underline{6}$. 
Despite these efforts and as discussed in the Results (Section 4), the agreement of the measurements was still limited by the stability of each aerosol source. This stability was independently quantified by bypassing the AAC in Figure $\underline{5}$ and using the CPC to measure the total number concentration $\left(N_{\text {tot }}\right)$ of each aerosol source over 2 hours. To operate the CPC in the same measurement mode during these stability measurements, the dilution ratio of the disk dilutor was increased to 3000,500 and 5000 for the DOS, $\mathrm{NaCl}$ and soot sources, respectively.

\section{Results and Discussion}

\subsection{Deconvolution of Size Distribution for Scanning AAC}

\subsubsection{Agreement with Steady-State AAC}

The agreement between the aerodynamic size distributions measured by stepping and scanning the AAC for all three aerosol sources (i.e. DOS, $\mathrm{NaCl}$ and soot particles) was generally within a few percent but did vary by up to $11.3 \%$, as shown in Figure 7 . This agreement was based on fitting 9 the parameters of a lognormal distribution to each of the measured size distributions, specifically the Count Median Diameter (CMD), Geometric Standard Deviation (GSD) and total number concentration of the particles $\left(N_{\text {tot }}\right)$.

However, the agreement between measurements of the steady-state and scanning AAC was limited by the stability of each aerosol source. As previously mentioned, the stability of each aerosol source was independently quantified by using the CPC to directly measure the total number concentration $\left(N_{\text {tot }}\right)$ of each aerosol source over 2 hours. The most stable source was DOS with a standard deviation of $0.8 \%$ and a maximum variation of $\pm 2.8 \%$ in its total number concentration of particles over 2 hours. This high stability resulted in the size distributions (i.e. CMD, GSD and $N_{\text {tot }}$ ) of DOS measured by the steady-state and scanning AAC agreeing within $2.0 \%$ or better. Furthermore, these agreements were consistent when operating the AAC at either low (LF: $Q_{\mathrm{a}}=0.3$ and $Q_{\mathrm{sh}}=3$ 
L/min) or high (HF: $Q_{\mathrm{a}}=1.5$ and $Q_{\mathrm{sh}}=15 \mathrm{~L} / \mathrm{min}$ ) classifier flows, as shown in Figures $\underline{7} \mathrm{a}$ and $\underline{7} \mathrm{~b}$, respectively.

After the 1 hour warm-up, the soot produced by the miniCAST had periods of relative stability (standard deviation of $4.0 \%$ in its total number concentration of particles over 2 hours). However, over 2 hours, its concentration shifted four times to a new mean that varied by greater than $10 \%$ from the previous one. These shifts occurred over $30 \mathrm{~s}$ or less, and therefore are hypothesized to be due to sudden changes in demand on the shared nitrogen, air and propane systems in the laboratory. However, no changes were noticed on the pressure gauges of the regulators. An example of this concentration shift occurred between the up and down scan of the steady-state AAC, as shown in Figure 7c. This result is independent of the scanning theory developed in this study, and further supports that the stability of the sources limited the agreement of the measurements. Despite this concentration shift, the CMD and GSD of the size distributions of the soot particles measured by the steady-state and scanning AAC agreed within $0.8 \%$ or better, as shown in Figure $7 \mathrm{c}$.

The mean number concentration of salt particles continuously shifted over 2 hours. This trend is reflected by a standard deviation of $16.2 \%$ in its total number concentration of particles over 2 hours, but a short-term standard deviation (i.e. based on a 600 s moving average) of only $1.5 \%$. This instability is likely due to the concentration of salt in the water of the Collison nebulizer changing over time from its atomization jet draining to its fluid reservoir. This trend agrees with the AAC results, whether they were collected via stepping or scanning the AAC, with each consecutive measurement of size distribution shifting slightly in amplitude. As shown in Figure $\underline{7 d}$, this source instability results in the largest disagreement between the measurements of the steady-state and scanning AAC of $11.3 \%,-5.7 \%$ and $-11.0 \%$ for the CMD, GSD and $N_{\text {tot }}$, respectively. 
Due to these stability limitations, consecutive measurements and their repeatability from the same polydispersed aerosol source are not reported by this study. This exclusion is intentional as the stability of these aerosol sources dominates this repeatability, and thus, is not representative of the agreement between the steady-state and scanning AAC measurements. To determine the repeatability of measurements using the scanning AAC, PSL particles of known sizes were measured multiple times, as discussed in the next section.

All of the measurements using the steady-state AAC were collected at 32 classes per decade (CPD). The DOS, $\mathrm{NaCl}$ and soot measurements using the scanning AAC were collected with scan times $\left(t_{\mathrm{sc}}\right)$ of $600 \mathrm{~s}, 630 \mathrm{~s}$ and $300 \mathrm{~s}$, respectively. Based on the particle size range of the scans, these scan times resulted in scan constants $\left(\tau_{\mathrm{sc}}\right)$ of $85.4 \mathrm{~s}, 125.6 \mathrm{~s}, 89.9 \mathrm{~s}$ and $107.5 \mathrm{~s}$ for the DOS LF, DOS HF, $\mathrm{NaCl}$ and soot measurements, respectively. Therefore, the scan time and associated CPD of the measurements of the three aerosol sources using the scanning AAC were 1.1 to 2.6 times faster 10 and 6.1 to 9.0 times higher 11 classes per decades than those of the steady-state AAC, as shown in Figure 7.

\subsubsection{Agreement with PSL Particles}

For further validation, six different sizes of PSL particles (from $100 \mathrm{~nm}$ to $2.02 \mu$ $\mathrm{m}$ ) were also measured with the scanning AAC. A minimum of two up scans and two down scans were collected at each size of PSL particles. Based on the known density of the PSL particles (i.e. $1050 \mathrm{~kg} / \mathrm{m}^{3}$ ) and the theory developed by Johnson et al. (2018a), the aerodynamic size distribution measured by the scanning AAC for each PSL particle size was converted to its equivalent mobility distribution. As shown in Figure 8, all of the measured CMDs agree within 8.7\% of the stated particle sizes or $5.7 \%$ if the uncertainty in the stated sizes is considered. The grey shaded area in Figure $\underline{8}$ illustrates these sizing uncertainties in PSL particles, while the error bars illustrate the repeatability 12 of the CMDs measured by the scanning AAC. The measured CMDs, including 
between the up and down scans, are highly repeatable (average of $0.2 \%$ ), thus validating the delay times determined by the UDA method.

The agreement of these PSL measurements is not only a function of the scanning inversion, but the uncertainty of the flow, speed and geometry of the classifier. Therefore, the PSL results are biased by these other sources of uncertainty and can be corrected for through calibration. For example, Tavakoli et al. (2014) and Kinney et al. (1991) accounted for these biases by determining the effective length of the AAC (prototype) and DMA classifier based on PSL measurements. These scanning PSL results are similar to those of Johnson et al. (2018b), which found the measurements of the steady-state AAC agreed within $4.7 \%$ for nine PSL particle sizes between $29 \mathrm{~nm}$ and $2.02 \mu \mathrm{m}$ and observed a similar positive bias.

It should be noted that the narrowness of the size distributions produced by the PSL particles may conflict with the inversion of the scanning AAC. Specifically, the assumption (as mentioned in Section 2.10 and discussed in SI Section S8) that the concentration of the aerosol is constant over the width of the average transfer function of the scanning AAC may not be valid. However, this assumption is also used in the inversion of other aerosol classifiers, such as the DMPS (Knutson, 1976), SMPS (Wang and Flagan, 1990) and steady-state AAC (Johnson et al., 2018a). Therefore, these classifiers also suffer from similar issues when measuring narrow size distributions, such as PSL particles. However for typical SMPS measurements, Stolzenburg and McMurry (2018) recently showed that although this assumption causes significant errors in the width (i.e. GSD) of the distribution measured, it only has a minor effect $(<1 \%)$ on the measured CMD and amplitude (i.e. $N_{\text {tot }}$ ). These conclusions likely also apply to the AAC given the results and understanding of previous studies that use the AAC to measure a narrow size distribution generated using PSL particles (Tavakoli et al., 2014; Johnson et al., 2018b) or by using another aerosol classifier in tandem (Tavakoli and Olfert, 2014; Johnson 
et al., 2018a, 2020). Therefore, this assumption within the inversion likely does not significantly affect the CMD measured by the steady-state or scanning AAC, including the PSL results shown in this study.

\subsection{Uniform versus Viscous Axial Flow}

Similar to the SMPS theory developed by Wang and Flagan (1990), all of the theory developed for the scanning AAC in this study assumes uniform flow in the axial direction of the classifier. This assumption is equivalent to assuming all of the particles have the same residence time in the classifier, as highlighted by Section 2.4. However, in reality, the axial flow in the AAC classifier is viscous and varies as a function of radial position due to no-slip boundary conditions at the walls of the classifier. Therefore, particles closer to the classifier walls will have lower axial velocities, and thus longer residence times. This varying velocity profile does not affect the steady-state transfer function of the DMA (Knutson and Whitby, 1975; Hoppel, 1978) or AAC (Tavakoli and Olfert, 2013). However, it does distort the transfer function of the SMPS as the ratio of the mean residence time of the particles in the classifier to the scan constant (i.e. $t_{\mathrm{f}} / \tau_{\mathrm{sc}}$ ) increases (Collins et al., 2004; Dubey and Dhaniyala, 2008; Mamakos et al., 2008). For example at $t_{\mathrm{f}} / \tau_{\mathrm{sc}}=3.11$, the median electrical mobility and particle counts (i.e. area under transfer function of DMA during scanning) measured by the SMPS vary by $46 \%$ and $55 \%$, respectively, from those predicted by assuming uniform axial flow (Mamakos et al., 2008). However as the $t_{\mathrm{f}}$ to $\tau_{\mathrm{sc}}$ ratio approaches zero, the transfer function of the SMPS accounting for viscous flow converges to that of the steady-state DMA (Collins et al., 2004; Dubey and Dhaniyala, 2008; Mamakos et al., 2008).

For this study, the experimental results of the polydispersed sources were collected with $t_{\mathrm{f}} / \tau_{\mathrm{sc}}=0.01$ to 0.06 , while those of the PSL particles were collected with $t_{\mathrm{f}} / \tau_{\mathrm{sc}}=0.01$ to 0.13 . Therefore, the effect of viscous axial flow on these results is likely insignificant. For reference, at a $t_{\mathrm{f}} / \tau_{\mathrm{sc}}$ of 0.16 the median electrical mobility and particle counts measured by the SMPS only shift by $2 \%$ or 
less (Collins et al., 2004; Mamakos et al., 2008). Furthermore, given the acceleration/deceleration limitations of the AAC classifier (as discussed in Section 2.3), typical AAC scans are less likely to achieve $t_{\mathrm{f}}$ to $\tau_{\mathrm{sc}}$ ratios where the effects of viscous axial flow need to be considered for measurement accuracy.

However, the $t_{\mathrm{f}}$ to $\tau_{\mathrm{sc}}$ ratios the AAC can achieve increase as its scanning range is narrowed or shifted to lower classifier speeds. For example, scanning the AAC based on its maximum ${ }^{5}$ acceleration/deceleration between 20 and $100 \mathrm{rad} / \mathrm{s}$ at low classifier flows $\left(Q_{\mathrm{a}}=0.3 \mathrm{~L} / \mathrm{min}\right.$ and $Q_{\mathrm{sh}}=3 \mathrm{~L} / \mathrm{min}$ ) results in $t_{\mathrm{f}} / \tau_{\mathrm{sc}}$ of 0.66 or 1.30 for the up or down scan, respectively. At standard gas conditions and a detector counting time $\left(t_{\mathrm{c}}\right)$ of $1 \mathrm{~s}$, this up scan measures the aerodynamic size distribution between 624 and $2433 \mathrm{~nm}$ particles at 19 CPD in $27 \mathrm{~s}$, while the down scan measures the aerodynamic size distribution between 798 and 2207 $\mathrm{nm}$ particles at $10 \mathrm{CPD}$ in $14 \mathrm{~s}$. These scan times (i.e. 27 and $14 \mathrm{~s}$ ) also approach the residence time of the particles in the classifier (i.e. $5.5 \mathrm{~s}$ ), which would likely further affect the measurements.

Therefore, the effect of viscous axial flow on the transfer function of the scanning AAC needs to be investigated in future work. Given the difference in classifier dimensions between the AAC and DMA, the threshold value for $t_{\mathrm{f}} / \tau_{\mathrm{sc}}$ that corresponds to appreciable effects of viscous axial flow is likely different between the instruments. Furthermore, some aspects of their classification methods are reversed. For example, increasing the total gas flow in the classifier shifts the measurement range to smaller particle sizes in the DMA, but larger particle sizes in the AAC. Also, continuously increasing the voltage in the DMA classifies progressively larger particles, while continuously increasing the speed of the AAC classifies progressively smaller particles. Therefore, the difference in distortions observed in the transfer function between the up and down scan of the SMPS at fast scan times (Collins et al., 2004; Dubey and 
Dhaniyala, 2008; Mamakos et al., 2008) may appear during the opposite scans of the AAC (i.e. during the down and up scan, respectively).

\subsection{Delay Time}

The delay times $\left(t_{\mathrm{d}}\right)$ of the scanning AAC were determined by minimizing the difference between consecutive up and down scans. This method is similar to the up-down agreement (UDA) approach described by Collins et al. (2004) for the SMPS. For the polydispersed aerosol measurements using the $3775 \mathrm{CPC}$, the delay times were $7 \mathrm{~s}$ and $-0.1 \mathrm{~s}$ at low and high flows, respectively. The slightly negative delay time at high-flow is due to internal software and communication timings between the AAC and CPC. For the PSL particle measurements using the $3752 \mathrm{CPC}$, the delay times varied from 6.9 to 9.7 and 3.0 to $4.2 \mathrm{~s}$ at low and high flows, respectively.

This variation in delay times for the PSL results is likely due to its scans being collected more quickly than the scans of the polydispersed aerosols (i.e. $\tau_{\mathrm{sc}}$ approximately halved), and assuming the delay time is a constant rather than a distribution (Collins et al., 2004). Viscous flow also occurs in the plumbing between the classifier outlet and the detection region of the particle detector. This flow profile smears the detection times of the particles classified at each measurement time $\left(t_{\mathrm{m}}\right)$. Russell et al. (1995) showed this effect is significant in the SMPS at high ratios of mixing time to scan constant $\left(\tau_{\mathrm{sc}}\right)$. Since the downstream plumbing and particle detector are the same in the SMPS or scanning AAC, previous theory for this smearing effect in the SMPS can be readily adapted to the scanning AAC.

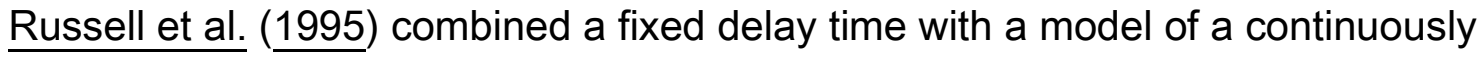
stirred tank reaction to represent the distribution of detection times and derive an "effective transfer function" for the DMA. Collins et al. (2002) found similar outcomes, but simplified the approach by assuming an exponential decay to remove the effect of the mixing within the detector first, then inverting the data 
based on the DMA response. Kanaparthi et al. (2018) also accounted for these smearing effects from downstream flows. They took a more comprehensive approach by incorporating these effects, as well as the impacts of the plumbing delay and counting times, directly into the arrival time transfer function of the SMPS. All three studies found as the ratio of mixing/smearing time (where higher values correspond to greater non-ideal flows) to scan constant increased, the size distributions measured by the SMPS were broader and had lower amplitudes.

Assuming a constant mixing time for a particular detector and flow rate, its ratio to the constant $\left(\tau_{\mathrm{sc}}\right)$ of each AAC scan varies by $188 \%$ and $76 \%$ for the PSL measurements at low and high flow, respectively. These variations are higher than the $26 \%$ observed in the same ratio for measurements of the polydispersed aerosols at low or high flow. Therefore, this higher variation likely drives the higher variance in the delay time of the PSL measurements. For example, the 6.9 $\mathrm{s}$ and $9.7 \mathrm{~s}$ delay times at low-flow correspond to $300 \mathrm{~s}$ and $90 \mathrm{~s}$ scans, respectively. If only $60 \mathrm{~s}$ scans of PSL at low-flow are considered, the variance in the mixing time to scan constant reduces by $34 \%$ and the variance in the delay time reduces by $60 \%$ ( 8.3 to $9.3 \mathrm{~s}$ ).

Assuming the same mixing time as Russell et al. (1995) and Collins et al. (2004) of $1.5 \mathrm{~s}$, its ratio to the scan constant varied from 0.012 to 0.036 for the experimental results in this study. Although this range is at the lower end of the 0.035 to 0.345 range tested by Collins et al. (2002) for the SMPS, they still observed a slight broadening of the distribution at 0.035 due to flow mixing downstream of the DMA. Therefore, it is unlikely this downstream flow effect had any appreciable impact on the measurements of the scanning AAC in this study. However, this effect still manifested in the variation of the delay times corresponding to the PSL measurements. 
This result supports the conclusion of Collins et al. (2004) that the delay time determined by the UDA approach masks the smearing effects of the downstream flow and thus deviates from the true delay time. If the downstream plumbing is minimized, the mixing time in the detector usually dominates and varies significantly between different detectors (Russell et al., 1995; Collins et al., 2002). Therefore, the delay time for the scanning AAC must be determined for any significant change in the scanning constant $\left(\tau_{\mathrm{sc}}\right)$, downstream plumbing, flow rate or particle detector. This requirement could be relaxed in the future by following similar approaches as those previously discussed (i.e. Russell et al., 1995, Collins et al., 2002, Kanaparthi et al., 2018) and accounting for the smearing effects of the flow downstream of the AAC within or before the data inversion. However, similar to the viscous flow effects in the classifier, additional testing is required with $A A C$ scans significantly faster than those in this study to amplify these flow effects and investigate their impact on the measurements. Computation fluid dynamic (CFD) simulations would also be valuable to verify the transfer function of the scanning AAC and capture the smearing effects from nonuniform axial flows, as well as the flow effects from the classifier inlet and outlet geometries.

\section{Conclusions and Summary}

To accelerate size distribution measurements using the AAC, this study develops the theory to continuously scan, rather than step, the AAC setpoint upstream of a particle detector. This theory shows that if the angular speed of the AAC is varied following an exponential function, the proportional change in the centrifugal force field over the residence time of particles in the classifier is independent of their inlet time. Based on this speed profile and assuming uniform axial flow, the transfer function of the scanning $A A C$ is derived by limited trajectory theory and found to be the same shape as that of the steady-state AAC. This outcome is used to adapt the parameterized transfer function of the steady-state AAC based on particle streamline theory, which accounts for non-idealized particle and flow 
behaviours within the classifier, to the scanning AAC. For both forms of the transfer function, the effect of the counting time of the particle detector downstream of the scanning AAC is quantified by determining the average transfer function over this time. The inversion of these average transfer functions is then determined, and the inversion parameter based on particle streamline theory and assuming uniform axial flow is found to be the same for either the steady-state or scanning AAC.

This scanning theory and its implementation are validated by the high agreement of the experimental results in this study. Specifically, the high agreement $(11.3 \%$ or better in terms of CMD, GSD and $N_{\text {tot }}$ ) of the measurements of the scanning $A A C$ compared to those of the steady-state AAC of three different aerosol sources (DOS, $\mathrm{NaCl}$ and Soot) with varying GSDs (1.3 to 2.7) and CMDs (66.4 $\mathrm{nm}$ to $292.1 \mathrm{~nm}$ ). If the stability of the aerosol source is considered, the agreement between the stepping and scanning measurements using the AAC improves to $2.0 \%$ or better. This validation is further supported by the high agreement (within $8.7 \%$ ) of scanning measurements of PSL particles (six sizes between $100 \mathrm{~nm}$ to $2.02 \mu \mathrm{m})$.

For the experimental results shown in this study, the measurements of the scanning AAC were 1.1 to 2.6 times faster and collected at 6.1 to 9.0 times higher CPD than those of the stepping AAC. This approach also leverages the high transmission efficiency and true monodispersity of the particles classified by the AAC. As a result, the measured distribution does not need to be corrected for multiply-charged particles, unlike those measured by electrostatic instruments, such as the SMPS or ELPI.

However, the minimum scan time of the AAC is limited by the acceleration/deceleration its classifier can achieve. Therefore, when measuring a similar size range (approximately 30 to $700 \mathrm{~nm}$ ), the up, down or combined scan of the $A A C$ is 3.2, 2.0 and 1.8 times longer, respectively, than the equivalent, 
standard SMPS scan, while at a similar or higher CPD (64 vs. 49 or 196). One key advantage of the scanning AAC is the broad range and upper limit of its particle sizing, scanning in ten minutes or less from approximately 30 to $2900 \mathrm{~nm}$ at low-flow and from approximately 200 to $6800 \mathrm{~nm}$ at high-flow. To further accelerate these measurements, future work could investigate if the transfer function of the AAC can be determined if the classifier is scanned at the maximum acceleration rather than scanned exponentially. However, it is likely that an analytical solution for this approach is not possible, and numerical methods would need to be used, significantly complicating the inversion process.

For typical AAC scans across broad size ranges, the assumption of uniform axial flow is likely valid given the maximum acceleration and deceleration the classifier can achieve. However, future work is required to consider the viscous flows within the classifier and downstream plumbing/detector, and as the scan time is reduced, quantify the smearing of the particle residence and detection times.

\section{Supplemental Material}

The supplemental material for this study can be accessed online. This material incorporates hyperlinks and bookmarks to further improve the navigation and interpretation of its contents.

\section{Acknowledgements}

This research would not have been possible without the support from Cambustion Ltd, The Rt. Hon. Sir Winston S. Churchill Society of Edmonton and C-FER Technologies.

\section{Notes}

1 Due to the APS operating outside the Stokes regime, where viscous forces dominate inertial forces (Kulkarni et al., 2011). 
2 The radial acceleration of the particle is neglected as the relaxation time of the particle is orders of magnitude smaller than the time over which the centrifugal force field changes. For example, the maximum particle relaxation time classified by the AAC is $140 \times 10^{-6} \mathrm{~s}$ (Cambustion, 2018) and the time constant $\left(\tau_{\mathrm{sc}}\right)$ of a typical AAC scan is 10's of seconds.

$\underline{3}$ This example assumes the aerosol is at standard conditions $(P=101.325 \mathrm{kPa}$ and $T=296.15 \mathrm{~K}$ ) and that the particles have an effective density of $1000 \mathrm{~kg} / \mathrm{m}^{3}$. This density results in the mobility and aerodynamic diameters of the particles being equivalent.

4The data inversion does not use assumptions, such as interpolation or averaging, to increase or decrease the CPD of the reported measurements.

5 These scan values correspond to the particle detector operating with a $1 \mathrm{~s}$ counting time $\left(t_{\mathrm{c}}\right)$, and the acceleration and deceleration capacity measured for AAC 2, as shown in Figure S2.2.

6 This estimate assumes the AAC is not spinning at the start of the down scan, and it takes approximately $140 \mathrm{~s}$ to reach the starting speed of $700 \mathrm{rad} / \mathrm{s}$.

7This ratio accounts for the standard $15 \mathrm{~s}$ retrace time for each consecutive SMPS scan.

8 This statement of independence neglects that the non-idealized parameters $\left(\lambda_{\Omega}\right.$ and $\mu_{\Omega}$ ) change based on the average setpoint of the scanning AAC over $t_{\mathrm{c}}$ (i.e. $\bar{\tau}_{\mathrm{sc}}^{*}$ as defined by Equation 46 ).

9The lognormal distribution was fitted to each measured size distributions using least-squares minimization.

10 This ratio is based on the average times $\left(t_{\mathrm{ss}}\right)$ to complete the up or down scan using the steady-state AAC. The down scan of the steady-state AAC was $1 \%$ to 
$8 \%$ faster than its up scan due to its higher capacity to decelerate than accelerate.

11 The CPD of the measurements collected using the scanning AAC also depend on the counting time $\left(t_{\mathrm{c}}\right)$ of the detector as shown in Equation 47. For this data, the CPC was operated with a counting time of $1 \mathrm{~s}$.

12 The repeatability was estimated assuming a $95 \%$ confidence interval and using a $t$-distribution.

\section{References}

Agarwal, J. K. and Sem, G. J. (1980). Continuous flow, single-particle-counting condensation nucleus counter. Journal of Aerosol Science, 11(4):343-357.

Babick, F., Hillemann, L., Stintz, M., Dillenburger, T., Pitz, M., Hellmann, A., Antonyuk, S., Ripperger, S., Huber, F. J. T., Will, S., Wernet, R., Seipenbusch, M., Gensch, M., Weber, A., Kiesler, D., Kruis, E., Friehmelt, R., and Sachweh, B. (2018). Multiparameter Characterization of Aerosols. Chemie Ingenieur Technik, 90(7):923-936.

Baron, P. A. (1986). Calibration and Use of the Aerodynamic Particle Sizer (APS 3300). Aerosol Science and Technology, 5(1):55-67.

Biskos, G., Mastorakos, E., and Collings, N. (2004). Monte-Carlo simulation of unipolar diffusion charging for spherical and non-spherical particles. Journal of Aerosol Science, 35(6):707-730.

Cambustion (2018). Aerodynamic Aerosol Classifier (AAC)- User Manual (Original Instructions) Version 1.13.

Chen, B. T., Cheng, Y. S., and Yeh, H. C. (1990). A Study of Density Effect and Droplet Deformation in the TSI Aerodynamic Particle Sizer. Aerosol Science and Technology, 12(2):278-285. 
Collins, D. R., Cocker, D. R., Flagan, R. C., and Seinfeld, J. H. (2004). The Scanning DMA Transfer Function. Aeroso/ Science and Technology, 38(8):833850.

Collins, D. R., Flagan, R. C., and Seinfeld, J. H. (2002). Improved Inversion of Scanning DMA Data. Aerosol Science and Technology, 36(1):1-9.

Dahneke, B. and Flachsbart, H. (1972). An aerosol beam spectrometer. Journal of Aerosol Science, 3(5):345-349.

Dubey, P. and Dhaniyala, S. (2008). Analysis of Scanning DMA Transfer Functions. Aerosol Science and Technology, 42(7):544-555.

Dubey, P. and Dhaniyala, S. (2011). A New Approach to Calculate Diffusional Transfer Functions of Scanning DMAs. Aerosol Science and Technology, 45(8):1031-1040.

Finlay, W. H. (2001). The mechanics of inhaled pharmaceutical aerosols: an introduction. Academic Press, San Diego.

Gopalakrishnan, R., Thajudeen, T., Ouyang, H., and Hogan, C. J. (2013). The unipolar diffusion charging of arbitrary shaped aerosol particles. Journal of Aerosol Science, 64(Supplement C):60-80.

Hinds, W. C. (1999). Aerosol technology: properties, behavior, and measurement of airborne particles. Wiley-Interscience Publication, Hoboken, NJ, 2nd ed. edition.

Hochrainer, D. (1971). A new centrifuge to measure the aerodynamic diameter of aerosol particles in the submicron range. Journal of Colloid and Interface Science, 36(2):191-194. 
Hoppel, W. A. (1978). Determination of the aerosol size distribution from the mobility distribution of the charged fraction of aerosols. Journal of Aerosol Science, 9(1):41-54.

Huang, Y., Seinfeld, J. H., and Flagan, R. C. (2020). Diffusional transfer function for the scanning electrical mobility spectrometer (SEMS). Aerosol Science and Technology, 54(10):1157-1168.

Jayne, J. T., Leard, D. C., Zhang, X., Davidovits, P., Smith, K. A., Kolb, C. E., and Worsnop, D. R. (2000). Development of an Aerosol Mass Spectrometer for Size and Composition Analysis of Submicron Particles. Aerosol Science and Technology, 33(1-2):49-70.

Johnson, T. J., Irwin, M., Symonds, J. P. R., Olfert, J.S., and Boies, A. M. (2018a). Measuring aerosol size distributions with the aerodynamic aerosol classifier. Aerosol Science and Technology, 52(6):655-665.

Johnson, T. J., Nishida, R. T., Irwin, M., Symonds, J. P. R., Olfert, J. S., and Boies, A. A. M. (2020). Measuring the bipolar charge distribution of nanoparticles: Review of methodologies and development using the Aerodynamic Aerosol Classifier. Journal of Aerosol Science, 143:105526.

Johnson, T. J., Nishida, R. T., Irwin, M., Symonds, J. P. R., Olfert, J. S., and Boies, A. M. (2018b). Agreement Between Different Aerosol Classifiers Using Spherical Particles. In Cambridge Particle Meeting, Cambridge, UK, June 15, 2018.

Kanaparthi, M. A., Cevaer, S. D., and Dhaniyala, S. (2018). Towards near realtime SEMS size distribution measurements under up-scan operation. Journal of Aerosol Science, 126:217-230.

Keskinen, J., Pietarinen, K., and Lehtimäki, M. (1992). Electrical low pressure impactor. Journal of Aerosol Science, 23(4):353-360. 
Kielser, D. and Kruis, F. (2017). High resolution measurement of aerodynamic focusing behaviour of nanoparticles in different gases and gas- mixtures using a Differential Aerodynamic Particle Sizer (DAPS). In 2017 European Aerosol Conference, Zurich. August 29, 2017. EAC.

Kinney, P. D., Pui, D. Y. H., Bryner, N. P., and Mulholland, G. W. (1991). Use of the electrostatic classification method to size 0.1 micrometer SRM particles - A feasibility study. Journal of Research of the National Institute of Standards and Technology, 96(2):147-176.

Knutson, E. O. (1976). Extended Electric Mobility Method for Measuring Aerosol Particle Size and Concentration. In LIU, B. Y. H., editor, Fine Particles, pages 739-762. Academic Press.

Knutson, E. O. and Whitby, K. T. (1975). Aerosol classification by electric mobility: apparatus, theory, and applications. Journal of Aerosol Science, 6(6):443-451.

Kulkarni, P., Baron, P. A., and Willeke, K., editors (2011). Aerosol measurement: principles, techniques, and applications. Wiley, Hoboken, N.J., 3rd ed. edition.

Liu, P., Ziemann, P. J., Kittelson, D. B., and McMurry, P. H. (1995). Generating Particle Beams of Controlled Dimensions and Divergence: I. Theory of Particle Motion in Aerodynamic Lenses and Nozzle Expansions. Aeroso/ Science and Technology, 22(3):293-313.

Mai, H. and Flagan, R. C. (2018). Scanning DMA Data Analysis I. Classification Transfer Function. Aerosol Science and Technology, 52(12):1382-1399.

Mai, H., Kong, W., Seinfeld, J. H., and Flagan, R. C. (2018). Scanning DMA data analysis II. Integrated DMA-CPC instrument response and data inversion. Aerosol Science and Technology, 52(12):1400-1414. 
Mamakos, A., Ntziachristos, L., and Samaras, Z. (2008). Differential mobility analyser transfer functions in scanning mode. Journal of Aerosol Science, 39(3):227-243.

Marple, V. A., Rubow, K. L., and Behm, S. M. (1991). A Microorifice Uniform Deposit Impactor (MOUDI): Description, Calibration, and Use. Aerosol Science and Technology, 14(4):434-446.

Mazumder, M. K. and Kirsch, K. J. (1977). Single particle aerodynamic relaxation time analyzer. Review of Scientific Instruments, 48(6):622-624.

Ouf, F.-X. and Sillon, P. (2009). Charging Efficiency of the Electrical Low Pressure Impactor's Corona Charger: Influence of the Fractal Morphology of Nanoparticle Aggregates and Uncertainty Analysis of Experimental Results. Aerosol Science and Technology, 43(7):685-698.

Rao, N. P., de la Mora, J. F., and McMurry, P. H. (1992). High-resolution aerodynamic spectrometry of submicron particles: sheathed variable-cut impactors vs other devices. Journal of Aerosol Science, 23(1):11-26.

Renninger, R. G., Mazumder, M. K., and Testerman, M. K. (1981). Particle sizing by electrical single particle aerodynamic relaxation time analyzer. Review of Scientific Instruments, 52(2):242-246.

Russell, L. M., Flagan, R. C., and Seinfeld, J. H. (1995). Asymmetric Instrument Response Resulting from Mixing Effects in Accelerated DMA-CPC Measurements. Aerosol Science and Technology, 23(4):491-509.

Saari, S., Arffman, A., Harra, J., Rönkkö, T., and Keskinen, J. (2018).

Performance evaluation of the HR-ELPI + inversion. Aerosol Science and Technology, 52(9):1037-1047. 
Stöber, W. and Flachsbart, H. (1971). High resolution aerodynamic size spectrometry of quasi-monodisperse latex spheres with a spiral centrifuge. Journal of Aerosol Science, 2(2):103-116.

Stolzenburg, M. R. and McMurry, P. H. (2008). Equations Governing Single and Tandem DMA Configurations and a New Lognormal Approximation to the Transfer Function. Aerosol Science and Technology, 42(6):421-432.

Stolzenburg, M. R. and McMurry, P. H. (2018). Accuracy of recovered moments for narrow mobility distributions obtained with commonly used inversion algorithms for mobility size spectrometers. Aerosol Science and Technology, 52(6):614-625.

Talukdar, S. S. and Swihart, M. T. (2003). An Improved Data Inversion Program for Obtaining Aerosol Size Distributions from Scanning Differential Mobility Analyzer Data. Aerosol Science and Technology, 37(2):145-161.

Tavakoli, F. and Olfert, J. S. (2013). An Instrument for the Classification of Aerosols by Particle Relaxation Time: Theoretical Models of the Aerodynamic Aerosol Classifier. Aerosol Science and Technology, 47(8):916-926.

Tavakoli, F. and Olfert, J. S. (2014). Determination of particle mass, effective density, mass-mobility exponent, and dynamic shape factor using an aerodynamic aerosol classifier and a differential mobility analyzer in tandem. Journal of Aerosol Science, 75:35-42.

Tavakoli, F., Symonds, J. P. R., and Olfert, J. S. (2014). Generation of a Monodisperse Size-Classified Aerosol Independent of Particle Charge. Aerosol Science and Technology, 48(3):i-iv.

TSI (2007). Model 3775 Condensation Particle Counter: Operation and Service Manual Revision D. 
TSI (2017). Condensation Particle Counter Model 3752- Operation Manual Revision B.

Wang, S. C. and Flagan, R. C. (1990). Scanning Electrical Mobility Spectrometer. Aerosol Science and Technology, 13(2):230-240.

Wilson, J. C. and Liu, B. Y. H. (1980). Aerodynamic particle size measurement by laser-doppler velocimetry. Journal of Aerosol Science, 11(2):139-150.

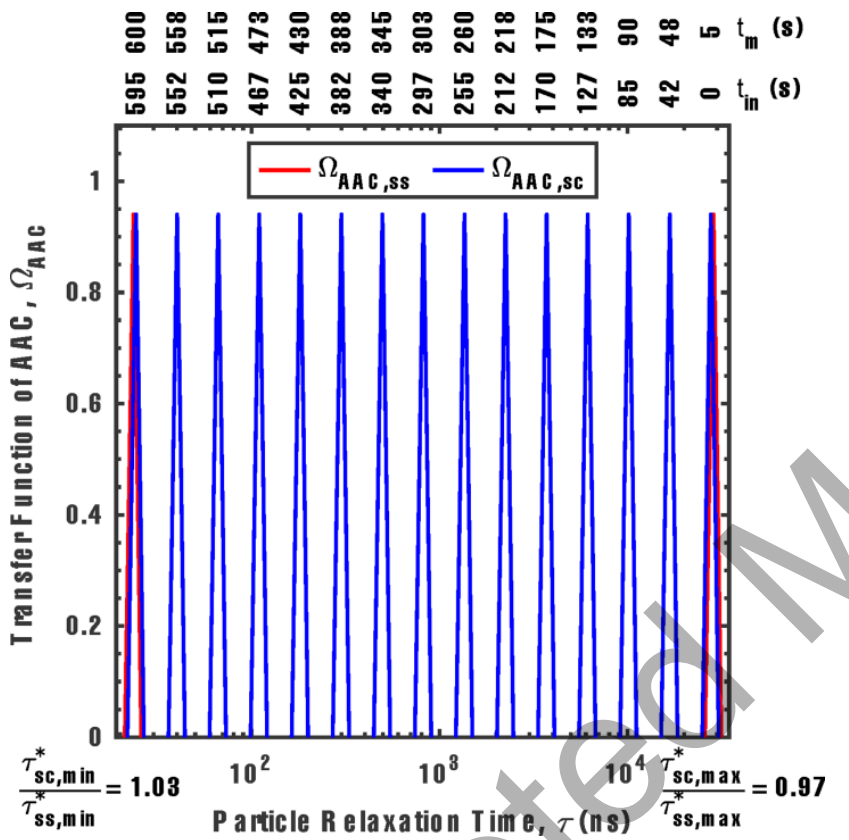

Fig. 1 Example of the transfer function $\left(\Omega_{\mathrm{AAC}}\right)$ of the steady-state (ss) or scanning (sc) AAC based on limited trajectory theory (as summarized in Table 1), where $\Omega_{\mathrm{AAC}, s c}$ is shown at 15 different times (in terms of $t_{\mathrm{in}}$ or $t_{\mathrm{m}}$ ) over a $600 \mathrm{~s}$ scan from $\omega_{\mathrm{s}}=20 \mathrm{rad} / \mathrm{s}$ to $\omega_{\mathrm{E}}=700 \mathrm{rad} / \mathrm{s}$, while $\Omega_{\mathrm{AAC}, \mathrm{ss}}$ is only shown at the start and end speeds of the scan (i.e. $\omega_{\mathrm{S}}$ and $\omega_{\mathrm{E}}$, respectively). 


$$
\begin{array}{|c|}
\hline \text { Idealized vs. N on-Idealized (NI) } \\
\hline \text { - Idealized }\left(\lambda_{\Omega}=1, \mu_{\Omega}=1\right) \\
- \text { - NI 1: } d_{\mathrm{a}, \mathrm{sc}}^{*}=30 \mathrm{~nm}, \tau_{\mathrm{sc}}^{*}=2.17 \mathrm{e}+01 \mathrm{~ns}, \lambda_{\Omega}=0.34, \mu_{\Omega}=0.45 \\
\cdots \text { NI 2: } \mathrm{d}_{\mathrm{a}, \mathrm{sc}}^{*}=300 \mathrm{~nm}, \tau_{\mathrm{sc}}^{*}=4.22 \mathrm{e}+02 \mathrm{~ns}, \lambda_{\Omega}=0.76, \mu_{\Omega}=0.55 \\
--\mathrm{N} \text { I 3: } \mathrm{d}_{\mathrm{a}, \mathrm{sc}}^{*}=3000 \mathrm{~nm}, \tau_{\mathrm{sc}}^{*}=2.87 \mathrm{e}+04 \mathrm{~ns}, \lambda_{\Omega}=0.79, \mu_{\Omega}=0.66 \\
\hline
\end{array}
$$

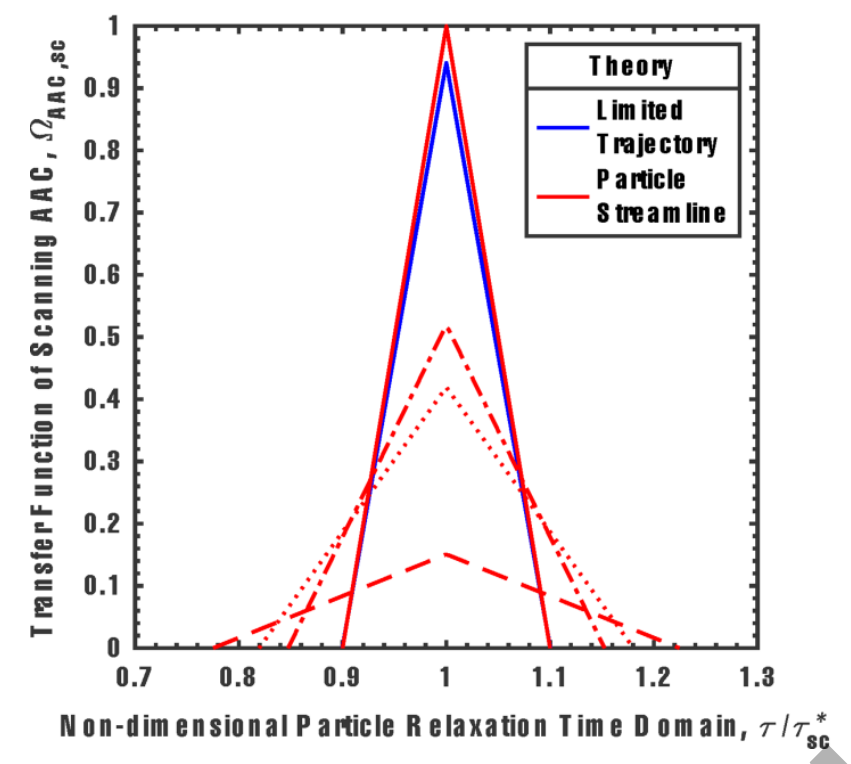

Fig. 2 Example comparing the transfer function of the Scanning AAC with balanced classifier flows based on limited trajectory and particle streamline theory. 


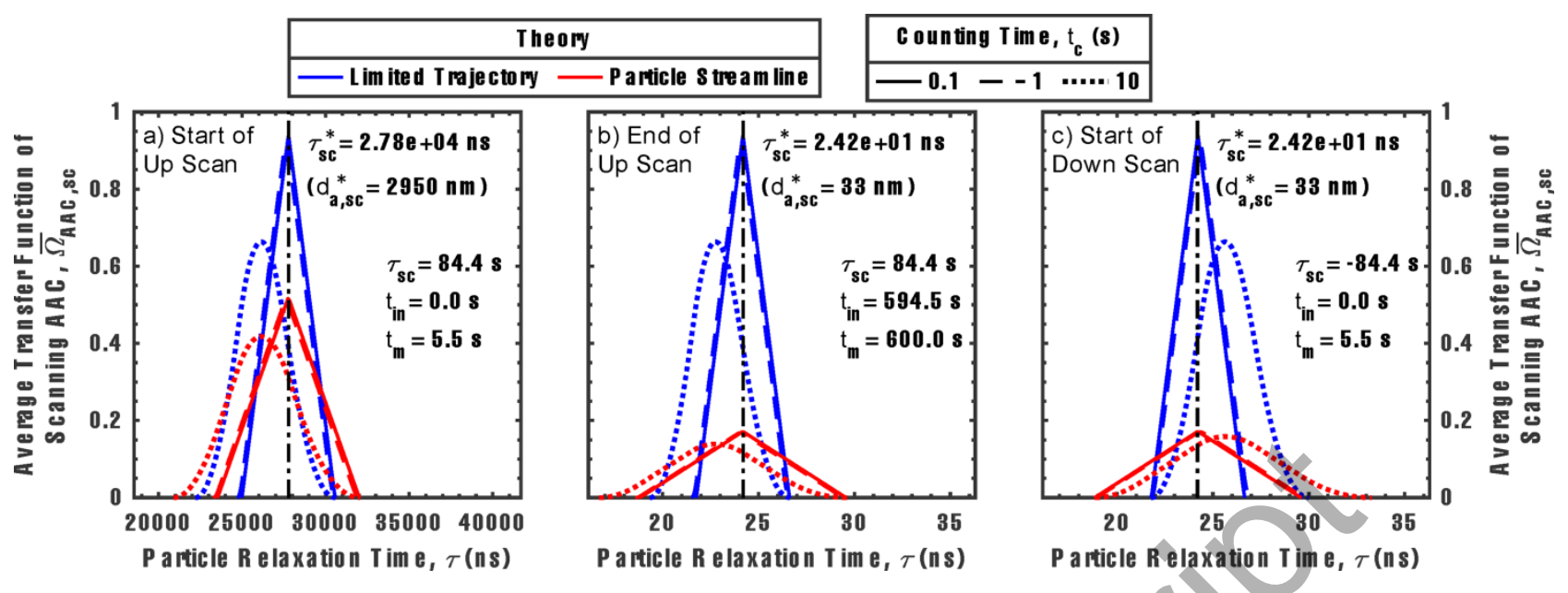

Fig. 3 The average scanning AAC transfer function $\Omega_{\text {AAC }}$ based on idealized limited trajectory or non-idealized particle streamline theory over three different particle detector counting times $\left(t_{c}\right)$, where subplots $\left.\left.a\right), b\right)$ and $c$ ) show the transfer functions at the start of the up scan, end of the up scan and start of the down scan, respectively. The vertical line in each subplot, denoted with a black, dash-dot line style, shows the instantaneous setpoint of the scanning AAC (in terms of relaxation time, $\tau_{\mathrm{sc}}^{*}$ or aerodynamic diameter $\left.{ }^{3}, d_{\mathrm{a}, \mathrm{sc}}^{*}\right)$ at the start of the counting interval of the particle detector. 


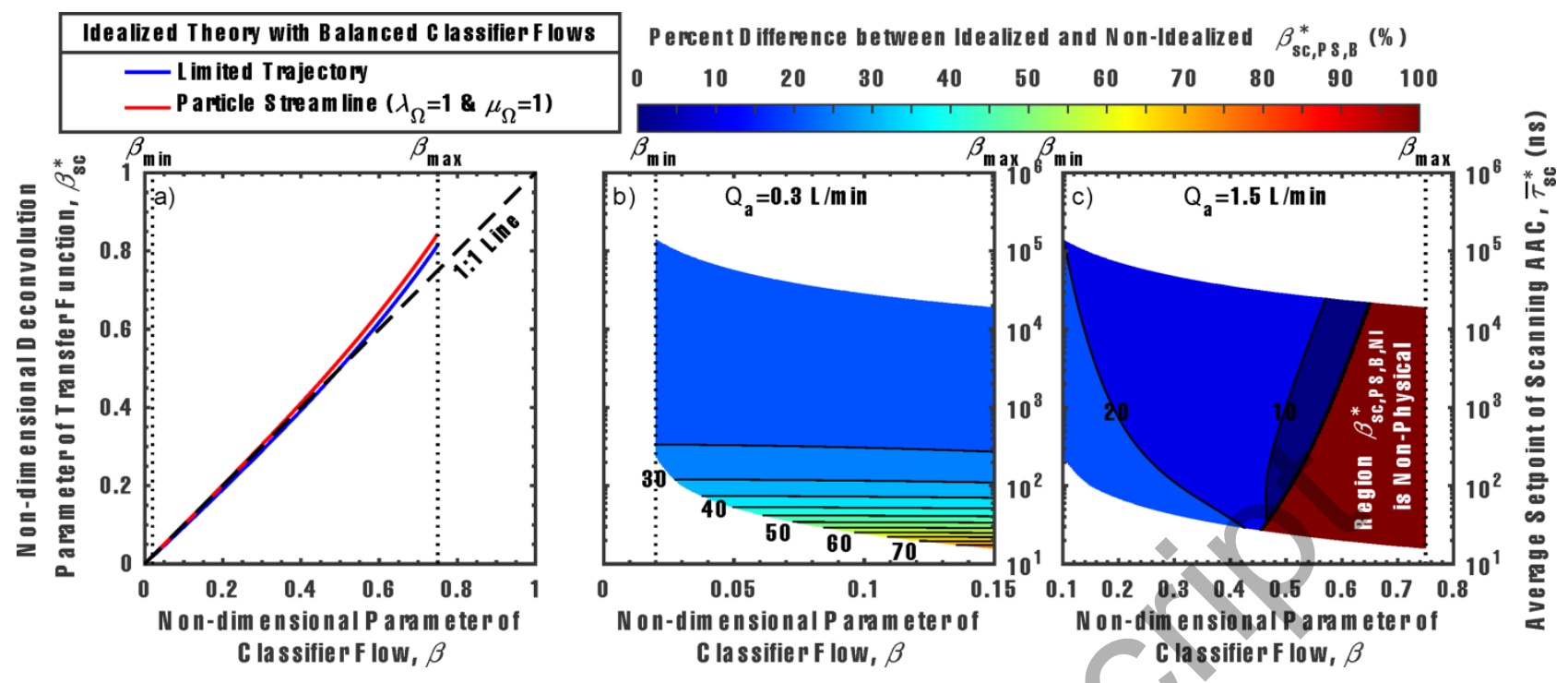

Fig. 4 A comparison of the non-dimensional deconvolution parameter $\left(\beta_{\mathrm{sc}}^{*}\right)$ of the transfer function of the scanning AAC, where subplot a) compares the parameters based on idealized limited trajectory or particle streamline theory, while subplots b) and c) compare the idealized and non-idealized ${ }^{3}$ deconvolution $^{-}$ parameters based on particle streamline theory at two sample flows $\left(Q_{\mathrm{a}}=0.3\right.$ or $1.5 \mathrm{~L} / \mathrm{min})$. 


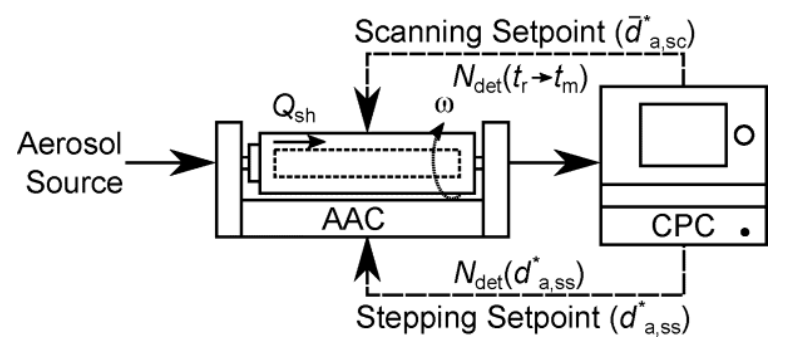

Fig. 5 Experimental setup used to validate deconvolution theory of scanning AAC. 
a) DOS Particles

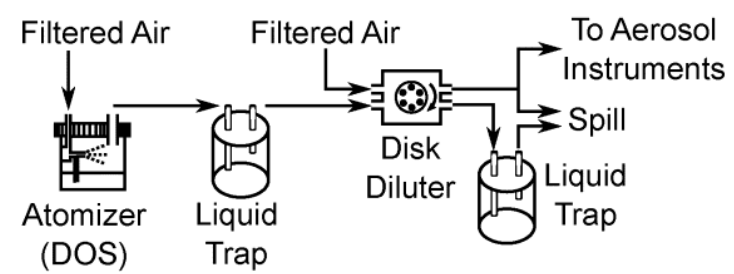

b) $\mathrm{NaCl}$ Particles

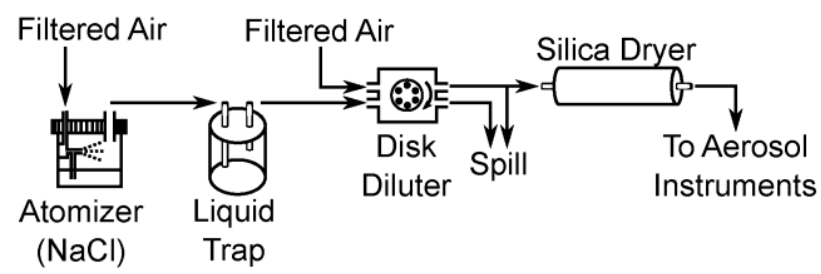

c) Soot Particles

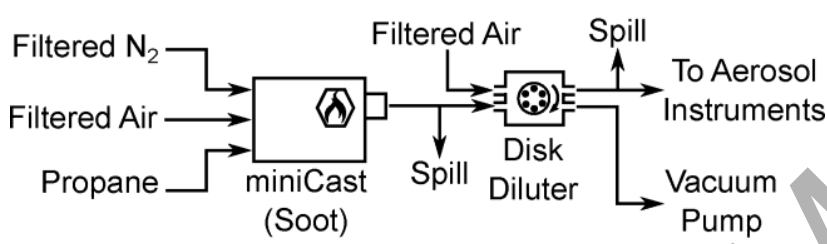

d) PSL Particles

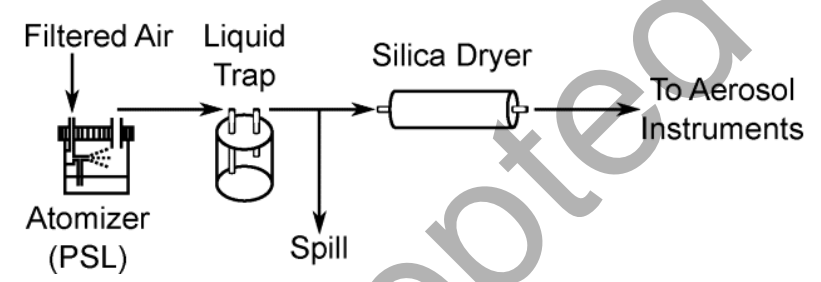

Fig. 6 The experimental setup used to generate each of the four aerosol sources used to validate the inversion theory of the scanning AAC. 

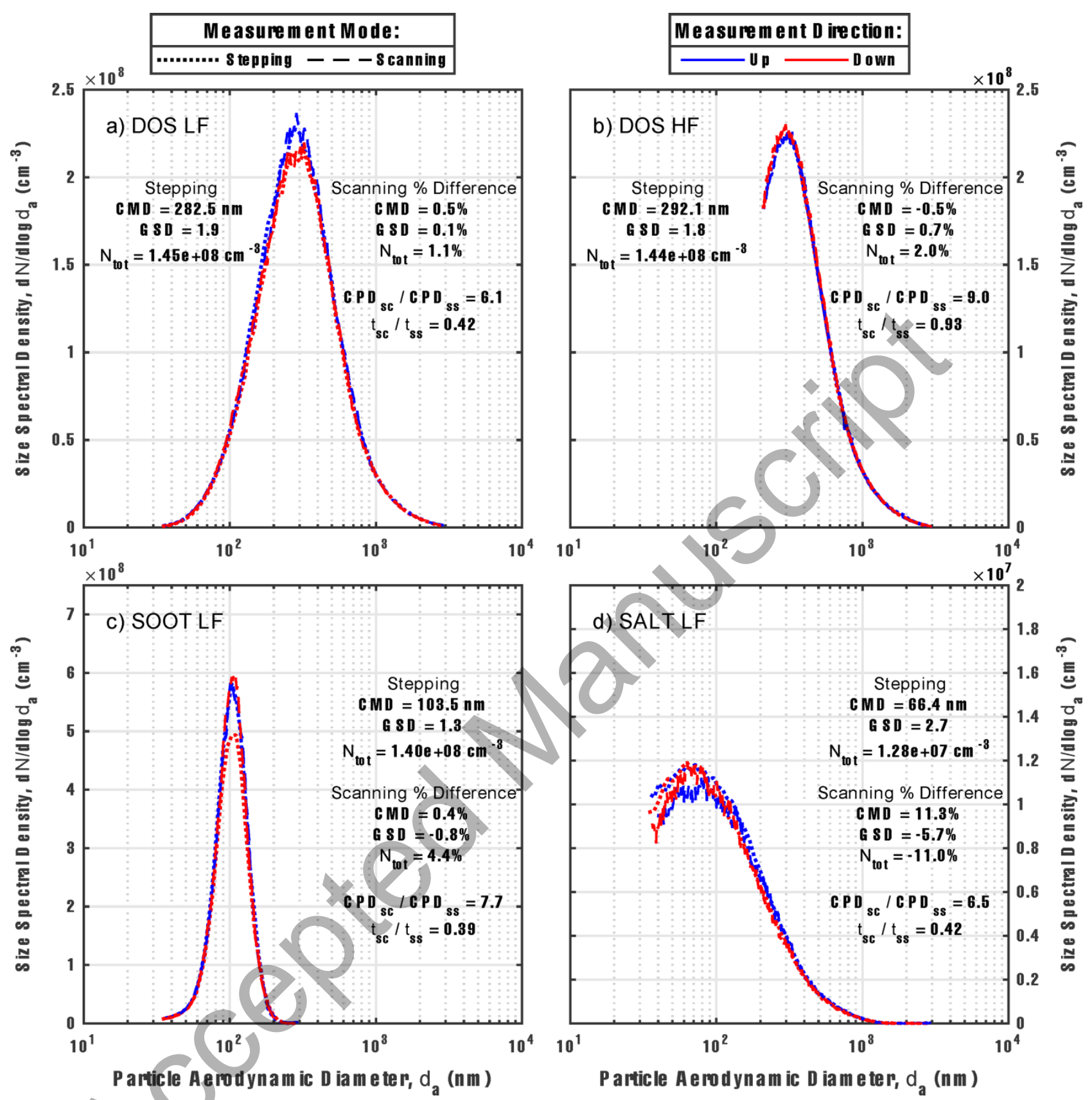

Fig. 7 Comparison of particle size distributions from different aerosol sources measured by stepping or scanning the AAC, where $a$ ) and b) show the AAC measurements of the DOS particle source at low and high classifier flows, respectively, while $\mathrm{c}$ ) and d) show the AAC measurements at low classifier flows of the soot and salt particle sources, respectively. 


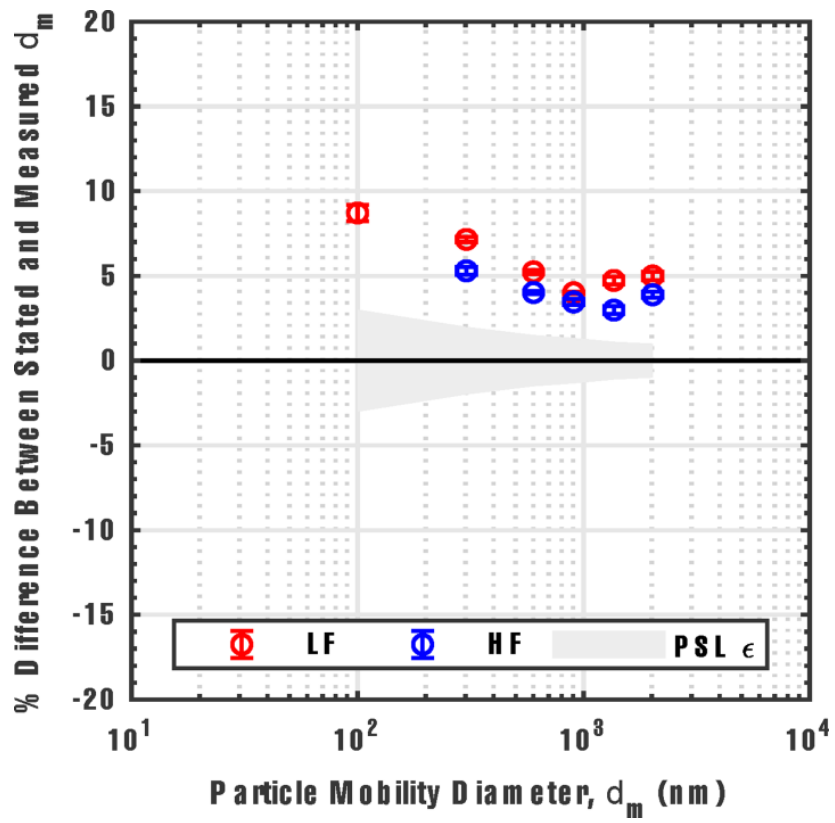

Fig. 8 Agreement between PSL calibration particles and the mobility equivalent CMD of the size distribution measured by the scanning AAC. The error bars depict the repeatability of the measurements (assuming a 95\% confidence interval), while the shaded region represents the uncertainty in the PSL sizes based on manufacturer specifications. 
Table 1 Transfer Function of the Steady-State (ss) or Scanning (sc) AAC based on Limited Trajectory (LT) Theory.

\begin{tabular}{|c|c|c|}
\hline Parameter & Equation & SI Eqn \# \\
\hline$\tau_{\max }$ & $\frac{1}{K} \ln \left(\frac{r_{2}}{r_{1}}\right)(20)$ & S3.5 \\
\hline$\tau_{\min }$ & $\frac{1}{2 K} \ln \left(\frac{Q_{\mathrm{a}}+Q_{\mathrm{sh}}-Q_{\mathrm{s}}\left(1-r_{1}^{2} / r_{2}^{2}\right)}{Q_{\mathrm{a}}+Q_{\mathrm{sh}}-Q_{\mathrm{sh}}\left(1-r_{1}^{2} / r_{2}^{2}\right)}\right)$ & \\
\hline$\tau^{*}$ & $\frac{\tau_{\min }+\tau_{\max }}{2}(22)$ & S3.9 \\
\hline$f_{1}$ & $\frac{Q_{\mathrm{a}}+Q_{\mathrm{sh}} r_{1}^{2} / r_{2}^{2}-\exp (-2 \tau K)\left[Q_{\mathrm{a}}+Q_{\mathrm{sh}}-Q_{\mathrm{s}}\left(1-r_{1}^{2} / r_{2}^{2}\right)\right]}{Q_{\mathrm{a}}\left(1-r_{1}^{2} / r_{2}^{2}\right)}$ (23) & S3.12 \\
\hline$f_{2}$ & $\frac{Q_{\mathrm{a}}+Q_{\mathrm{sh}}}{Q_{\mathrm{a}}}\left(\frac{\exp (-2 \tau K)-r_{1}^{2} / r_{2}^{2}}{1-r_{1}^{2} / r_{2}^{2}}\right)$ & S3.15 \\
\hline$f_{3}$ & $\frac{Q_{\mathrm{s}}}{Q_{\mathrm{a}}}(25)$ & S3.16 \\
\hline$\Omega_{\mathrm{AAC}}$ & $\max \left[0, \min \left(f_{1}, f_{2}, f_{3}, 1\right)\right](26)$ & S3.17 \\
\hline
\end{tabular}

Where $K$ equals $K_{\text {ss }}$ (Equation 16) or $K_{\text {sc }}$ (Equation 19) for the steady-state or scanning AAC, respectively. 
Table 2 Transfer Function of the Scanning AAC based on Particle Streamline (PS) Theory with Balanced Classifier Flows or Limited Trajectory (LT) Theory.

\begin{tabular}{|c|c|c|c|c|}
\hline $\begin{array}{l}\text { Paramet } \\
\text { er }\end{array}$ & Limited Trajectory (LT) & $\begin{array}{c}\text { Particle Streamline (PS) } \\
\text { with }\end{array}$ & \multicolumn{2}{|c|}{ SI Eqn \# } \\
\hline & & $\begin{array}{l}\text { Balanced (B) Classifier } \\
\text { Flows }\end{array}$ & & PS \\
\hline$\Omega_{\mathrm{AAC}, \mathrm{sc}}$ & $\max \left[0, \min \left(f_{1}, f_{2}, f_{3}, 1\right)\right](27)$ & $\mid \begin{array}{l}\mid \frac{\lambda_{\Omega} \mu_{\Omega}^{2}}{2 \beta}\left[\left|\frac{\tau}{\tau_{\mathrm{sc}, \mathrm{B}}^{*}}-\left(1+\frac{\beta}{\mu_{\Omega}}\right)\right|\right. \\
+\left|\frac{\tau}{\tau_{\mathrm{sc}, \mathrm{B}}^{*}}-\left(1-\frac{\beta}{\mu_{\Omega}}\right)\right|-2 \mid \frac{\tau}{\tau_{\mathrm{sc}, \mathrm{B}}^{*}}- \\
(28)\end{array}$ & $\$ 33.1$ & 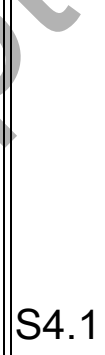 \\
\hline$\tau_{\mathrm{sc}}^{*}$ & & 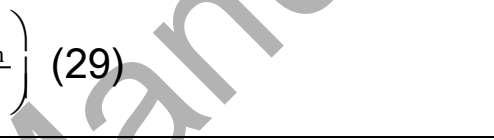 & $\begin{array}{l}S 3.3 \\
4\end{array}$ & $\begin{array}{l}S 4.1 \\
0\end{array}$ \\
\hline$c_{\tau^{*}}$ & $\begin{array}{l}\frac{1}{2 c_{\mathrm{sc}}} \ln \left(\frac{r_{2}}{r_{1}}\right) \\
\quad+\frac{1}{2} \ln \left(\frac{Q_{\mathrm{a}}+Q_{\mathrm{sh}}-Q_{\mathrm{s}}\left(1-r_{1}^{2}\right.}{Q_{\mathrm{a}}+Q_{\mathrm{sh}}-Q_{\mathrm{sh}}\left(1-r_{1}^{2}\right.}\right. \\
(30)\end{array}$ & $\frac{1}{c_{\mathrm{sc}}} \frac{2\left(r_{2}^{2}-r_{1}^{2}\right)}{(\beta+1)\left(r_{1}+r_{2}\right)^{2}}$ & $\begin{array}{l}\text { S3.3 } \\
6\end{array}$ & $\begin{array}{l}S 4.1 \\
1\end{array}$ \\
\hline$c_{\mathrm{sc}}$ & 8 & $\left.\left.\frac{-t_{\mathrm{f}}}{\tau_{\mathrm{sc}}}\right)\right](32)$ & $\begin{array}{l}S 3.2 \\
9\end{array}$ & $\begin{array}{l}S 4.1 \\
2\end{array}$ \\
\hline
\end{tabular}




\section{Table 3 Average Transfer Function of Scanning AAC over Counting Time of the Particle Detector.}

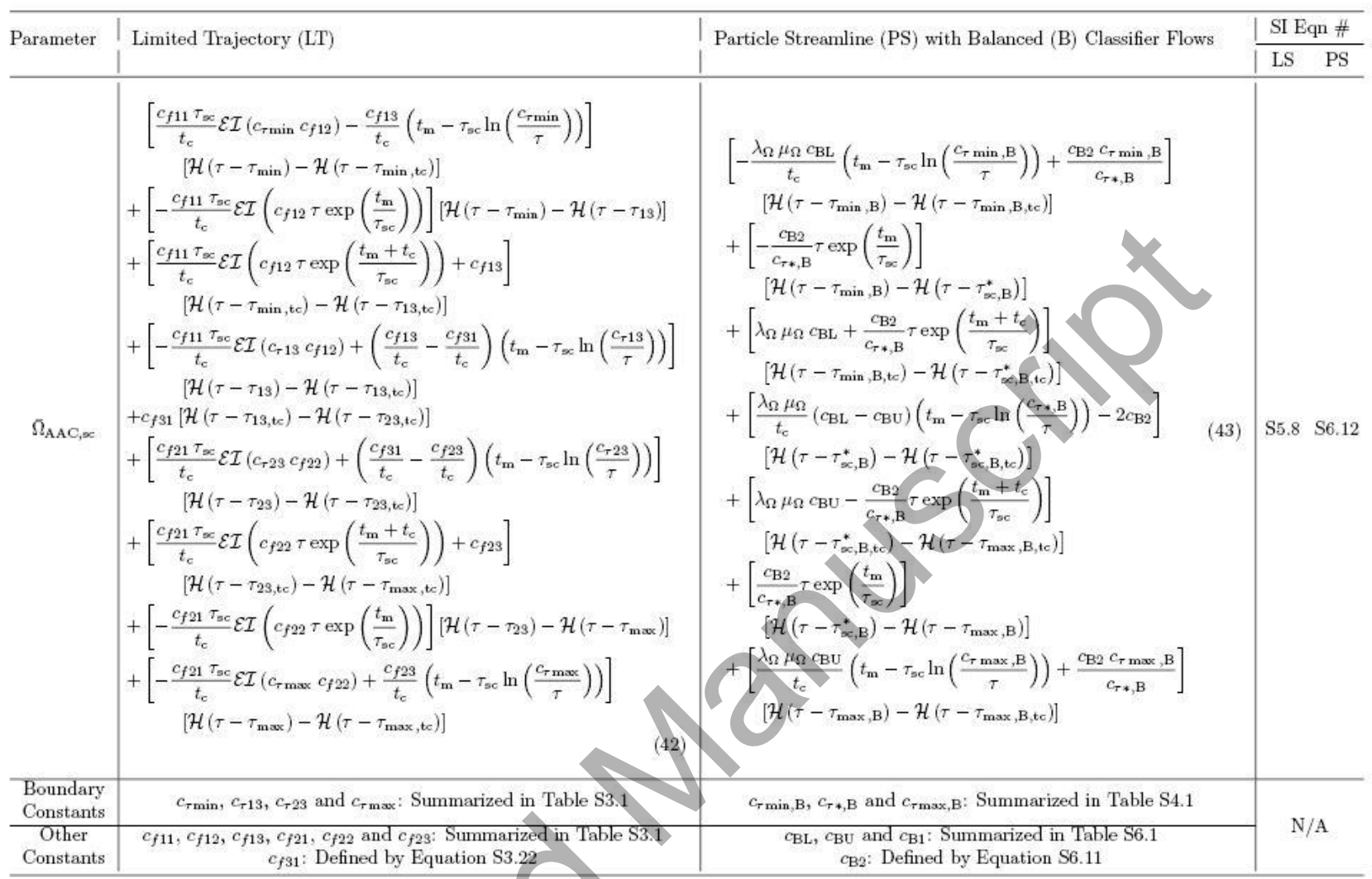

Where $\mathcal{H}(x-a)$ and $\mathcal{E I}(x)$ are the Heaviside and Exponential Integral Functions defined by Equations 35 and S5.17, respectively. 
Table 4 Parameters of the Scanning AAC.

\begin{tabular}{|c|c|c|c|}
\hline Parameter & \multicolumn{2}{|l|}{ Equation } & SI Eqn \# \\
\hline $\bar{\tau}_{\mathrm{sc}}^{*}$ & $\frac{-c_{\tau^{*}} \tau_{\mathrm{sc}}}{t_{\mathrm{c}}}\left[\exp \left(\frac{-\left(t_{\mathrm{m}}+t_{\mathrm{c}}\right)}{\tau_{\mathrm{sc}}}\right)-\exp (\right.$ & $\left.\left.\frac{-t_{\mathrm{m}}}{\tau_{\mathrm{sc}}}\right)\right]$ (46) & S7.4 \\
\hline $\mathrm{CPD}_{\mathrm{sc}}$ & $\frac{1}{\left|\log \left(\exp \left(\frac{-t_{\mathrm{c}}}{\tau_{\mathrm{sc}}}\right)\right)\right|}(47)$ & & S7.7 \\
\hline$\left.\frac{\bar{\tau}_{\mathrm{sc}}^{*}}{\tau_{\mathrm{ss}}^{*}}\right|_{\mathrm{s}}$ & $\frac{-t_{\mathrm{f}}}{t_{\mathrm{c}}\left[\exp \left(\frac{t_{\mathrm{f}}}{\tau_{\mathrm{sc}}}\right)-1\right]}\left[\exp \left(\frac{-t_{\mathrm{c}}}{\tau_{\mathrm{sc}}}\right)-1\right]$ & (48) & \\
\hline$\left.\bar{\tau}_{\mathrm{ss}}^{*}\right|_{\mathrm{s}}$ & $\omega_{\mathrm{S}}^{2} t_{\mathrm{c}} \exp \left(\frac{t_{\mathrm{sc}}-t_{\mathrm{f}}}{\tau_{\mathrm{sc}}}\right)\left[\exp \left(\frac{t_{\mathrm{f}}}{\tau_{\mathrm{sc}}}\right)-1\right.$ & & \\
\hline
\end{tabular}

Where $c_{\tau^{*}}$ equals Equation 30 or Equation 31 for the scanning AAC based on limited trajectory theory or particle streamline theory operating with balanced classifier flows, respectively. 
Table 5 Parameters of the AAC during Example Scans.

\begin{tabular}{|c|c|c|c|c|c|c|c|c|c|c|c|c|c|c|}
\hline \multirow[t]{3}{*}{ Flow } & \multirow[t]{3}{*}{ Scan } & \multicolumn{2}{|c|}{$\begin{array}{l}\text { Speed } \\
(\mathrm{rad} / \mathrm{s})\end{array}$} & \multirow[t]{3}{*}{\begin{tabular}{||c|} 
Min Scan \\
Time $^{5}, t_{\mathrm{sc}}(\mathrm{s})$
\end{tabular}} & \multicolumn{5}{|c|}{$\begin{array}{c}\begin{array}{c}\text { Scan } \\
\text { Constant, } \tau_{\mathrm{sc}}\end{array} \\
\text { (s) }\end{array}$} & \multicolumn{4}{|c|}{ Range $^{3,5}$, of Scanning } & \multirow[t]{3}{*}{$\begin{array}{c}\text { Classes per } \\
\text { Decade }^{5}, \mathrm{CPD}_{\mathrm{sc}}\end{array}$} \\
\hline & & \multirow[b]{2}{*}{$\omega_{\mathrm{S}}$} & \multirow[b]{2}{*}{$\omega_{\mathrm{E}}$} & & \multirow{3}{*}{85.3} & \multirow[b]{2}{*}{ Min } & \multirow{2}{*}{ Max } & \multicolumn{2}{|c|}{$d_{\mathrm{a}, \mathrm{ss}}^{*}(\mathrm{~nm})$} & \multicolumn{2}{|c|}{$\bar{\tau}_{\mathrm{sc}}^{*}(\mathrm{~ns})$} & \multicolumn{2}{|c|}{$\bar{d}_{\mathrm{a}, \mathrm{sc}}^{*}(\mathrm{~nm})$} & \\
\hline & & & & & & & & Min & Max & Start & End & Start & End & \\
\hline \multirow[t]{2}{*}{ LF } & Up & 20 & 700 & 607 & & 23 & 28708 & 32 & 3000 & 27637 & 24 & 2942 & 33 & $\mid 196$ \\
\hline & Down & 700 & 20 & 153 & -21.5 & & & & & 27 & 25808 & 37 & 2840 & 49 \\
\hline \multirow[t]{2}{*}{$\mathrm{HF}$} & Up & 20 & 500 & 333 & 51.7 & 230 & 143541 & 203 & 6803 & 140667 & 230 & 6734 & 203 & 119 \\
\hline & Down & 500 & 20 & 107 & -16.7 & & & & & 245 & 143143 & 211 & 6793 & 38 \\
\hline \multirow[t]{2}{*}{ LF } & Up & 76 & 700 & 379 & 85.3 & 23 & 1988 & 32 & 735 & 1914 & 24 & 720 & 33 & |196 \\
\hline & Down & 700 & 76 & 95 & -21.5 & & & & & 27 & 1787 & 37 & 693 & 49 \\
\hline
\end{tabular}

Where low-flow (LF) corresponds to 0.3 and $3 \mathrm{~L} / \mathrm{min}$ sample and sheath flow (i.e. $Q_{\mathrm{a}}$ and $Q_{\mathrm{sh}}$ ), respectively; high-flow $(\mathrm{HF})$ corresponds to 1.5 and $15 \mathrm{~L} / \mathrm{min}$ sample and sheath flow (i.e. $Q_{\mathrm{a}}$ and $Q_{\mathrm{sh}}$ ), respectively; and the corresponding residence time $\left(t_{\mathrm{f}}\right)$ of the particles in the classifier is 5.5 and $1.1 \mathrm{~s}$ for LF and HF, respectively. 
Table 6 Deconvolution of the Transfer Function of the Scanning AAC.

\begin{tabular}{|c|c|c|c|}
\hline Parameter & Limited Trajectory (LT) & $\begin{array}{l}\text { Particle Streamline } \\
\text { (PS) with }\end{array}$ & SI Eqn \# \\
\hline & & \begin{tabular}{|l} 
Balanced (B) \\
Classifier Flows
\end{tabular} & \begin{tabular}{|l||l} 
LT & PS
\end{tabular} \\
\hline$\| \beta_{\mathrm{sc}}^{*}$ & 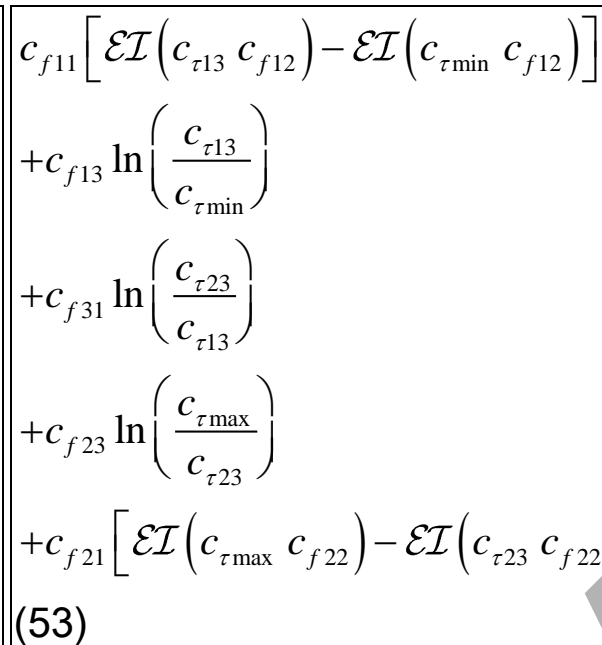 & $+\frac{\mu_{\Omega}}{\beta} \ln \left(1-\left(\frac{\beta}{\mu_{\Omega}}\right)^{2}\right.$ & $\mid$ S9.5 $\mathrm{S} 10.7$ \\
\hline
\end{tabular}

Where $\mathcal{E} \mathcal{I}(x)$ is the Exponential Integral Function defined by Equation S5.17, and the boundary constants $\left(c_{\text {mmin }}, c_{\tau 13}, c_{\tau 23}\right.$ and $c_{\text {tmax }}$ ) and other constants ( $c_{f 11}, c_{f 12}, c_{f 13}, c_{f 21}, c_{f 22}$ and $\left.c_{f 23}\right)$ for $\beta_{\mathrm{sc}}^{*}$ based on limited trajectory theory are defined in Table S3.1 and by Equation S3.22 $\left(c_{f 31}\right)$. 